\title{
Spin-four $\mathcal{N}=7 \mathrm{~W}$-supergravity: S-fold and double copy construction
}

\author{
Sergio Ferrara ${ }^{a, b, c}$ and Dieter Lüst ${ }^{a, d, e}$ \\ ${ }^{a}$ CERN, Theory Department, \\ 1211 Geneva 23, Switzerland \\ ${ }^{b}$ INFN - Laboratori Nazionali di Frascati, \\ Via Enrico Fermi 40, 00044 Frascati, Italy \\ ${ }^{c}$ Department of Physics and Astronomy and \\ Mani L. Bhaumik Institute for Theoretical Physics, U.C.L.A, \\ Los Angeles CA 90095-1547, U.S.A. \\ ${ }^{d}$ Arnold-Sommerfeld-Center for Theoretical Physics, \\ Ludwig-Maximilians-Universität, 80333 München, Germany \\ e Max-Planck-Institut für Physik, Werner-Heisenberg-Institut, \\ 80805 München, Germany \\ E-mail: sergio.ferrara@cern.ch, dieter.luest@lmu.de
}

ABSTRACT: In the present investigation we consider the possibility of having new massive, higher spin W-supergravity theories, which do not exist as four-dimensional perturbative models. These theories are based on a double copy construction of two supersymmetric field theories, where at least one factor is given by a $\mathcal{N}=3$ field theory, which is a nonperturbative S-fold of $\mathcal{N}=4$ super Yang-Mills theory. In this way, we can obtain as S-folds a new $\mathcal{N}=7$ (corresponding to 28 supercharges) W-supergravity and its $\mathcal{N}=7 \mathrm{~W}$ superstring counterpart, which both do not exist as four-dimensional perturbative models with an (effective) Langrangian description. The resulting field resp. string theory does not contain any massless states, but instead a massive higher spin-four supermultiplet of the $\mathcal{N}=7$ supersymmetry algebra. Furthermore we also construct a four-dimensional heterotic S-fold with $\mathcal{N}=3$ supersymmetry. It again does not exist as perturbative heterotic string model and can be considered as the heterotic counterpart of the $\mathcal{N}=3$ superconformal field theories, which were previously constructed in the context of type I orientfold models.

Keywords: Higher Spin Symmetry, String Duality, Superstrings and Heterotic Strings, Supersymmetric Gauge Theory

ARXIV EPRINT: 1805.10022 


\section{Contents}

1 Introduction 1

2 Double copy construction of massive W-supergravities 4

2.1 Double copy constructions 4

2.1.1 Standard massless supergravities as double copy constructions 5

$2.24 \mathrm{D}$ massive supermultiplets of $\mathcal{N}$-extended supersymmetry: 6

2.3 The $\mathcal{N}=3$ S-fold $\quad 7$

2.4 Non-standard massive W-supergravities as double copy constructions $\quad 10$

2.5 Massive $\mathcal{N}=7 \mathrm{~W}$-supergravity 11

3 String S-fold construction - Implementation of R-symmetry and Sduality transformations in heterotic and type II string theory 12

$\begin{array}{lll}3.1 & \text { R-symmetry and T-duality twist } & 16\end{array}$

$\begin{array}{lll}3.2 & \text { S-duality twist } & 18\end{array}$

4 Massive spectrum of W-superstrings $\quad 21$

4.1 Massive states of the unprojected four-dimensional fermionic string with $\mathcal{N}=4$ supersymmetry

4.2 Massive states of the $\mathbb{Z}_{4}$-projected four-dimensional fermionic string with $\mathcal{N}=3$ supersymmetry 23

4.3 Massive states of the bosonic string 23

$4.4 \mathcal{N}=3$ heterotic W-superstring 24

$4.5 \mathcal{N}=7$ type II W-superstring 24

5 Conclusions and outlook $\quad 25$

$\begin{array}{ll}\text { A Supercharges } & 27\end{array}$

\section{Introduction}

For space-dimensions $D \geq 4$ supergravity theories [1,2] have been investigated in great detail (for overviews see e.g. [3, 4]). Moreover this discussion has been extended in [5], where also a full classification of all possible number of supercharges has been obtained for the case of $D=3 .{ }^{1}$ One of the main results is that a supergravity theory with 28 supercharges, here commonly referred as $\mathcal{N}=7$ supergravity using four-dimensional language, is not possible. In fact, when constructing the corresponding superalgebras and also using CPT symmetry, the local $\mathcal{N}=7$ supersymmetry is always automatically extended to the case of maximal

\footnotetext{
${ }^{1}$ Recently, two-dimensional string constructions with exotic supersymmetries were constructed in [6].
} 
supersymmetry, namely to $\mathcal{N}=8$ supergravity with 32 supercharges. The absence of a perturbative $\mathcal{N}=7$ supergravity theory is analogous to the absence of any perturbative, four-dimensional supersymmetric gauge theories with rigid $\mathcal{N}=3$ supersymmetry. Again, using same kind of arguments as in supergravity, one can show that the $\mathcal{N}=3$ superalgebra of a CPT invariant gauge theory action is always extended to the maximal case of $\mathcal{N}=4$ rigid supersymmetry. To summarize, the three main assumptions, which enter the $\mathcal{N}=7$ supergravity as well as the $\mathcal{N}=3$ field theory no-go theorems, are as follows:

- The theory is weakly coupled.

- There exist a CPT invariant Langrangian description.

- For the case of supergravity, there exist a massless, N-extended spin-two supergravity multiplet and no higher spin massless multiplets.

The prime goal of this paper is to provide some good arguments for the existence of a new kind of $\mathcal{N}=7$ locally supersymmetric theory in $D=4$, which is not based on a conventional, perturbative supergravity action, but which however is strongly coupled and does not exists as a Langrangian theory. It will not contain a massless spin-two $\mathcal{N}=7$ supergravity multiplet, but a massive $\mathcal{N}=7$ multiplet of higher spin-four, which realizes the $\mathcal{N}=7$ supersymmetry algebra. In analogy to higher spin theories in two dimensions we call these theories $\mathcal{N}=7 \mathrm{~W}$-supergravity theories. ${ }^{2}$ In this way the above assumptions for the no-go theorems are circumvented. This new $\mathcal{N}=7 \mathrm{~W}$-supergravity theory is analogous to the $\mathcal{N}=3$ supersymmetric gauge theories, which were recently constructed in [7-9] and which are non-perturbative in the sense that they only exist at strong gauge coupling and that they also do not allow for a known Lagrangian formulation with a massless gauge supermultiplet. Furthermore they are conjectured to be superconformal. The $\mathcal{N}=3$ supersymmetric gauge theories can be constructed as S-folds starting from $\mathcal{N}=4$ supersymmetric Yang-Mills gauge theory and projecting on those operators, which are invariant under a combined action of R-symmetry and S-duality:

$$
\text { Field theory } \mathrm{S}-\text { fold : } \quad\left(\mathcal{N}=3_{\mathrm{SYM}}\right) \equiv\left(\mathcal{N}=4_{\mathrm{SYM}}\right) /(R \times S) \text {. }
$$

A closely related, holographically dual $\mathcal{N}=6$ supergravity in $A d S_{5}$ space was already constructed in [10], with the same kind of S-fold projection being used as in the $\mathcal{N}=3$ supersymmetric gauge theories of [7-9].

In the first part of the paper we will use the compelling observation that Einstein gravity can be regarded as the square of two Yang-Mills theries. This is the socalled double copy construction of (super)gravity theories [11-13], which was utilized for

$$
\mathcal{N}=8 \text { Sugra } \equiv(\mathcal{N}=4 \mathrm{YM})^{2}
$$

to compute scattering amplitudes in $\mathcal{N}=8$ supergravity from $\mathcal{N}=4$ super Yang-Mills amplitudes. The double copy construction was also used to obtain all possible standard

\footnotetext{
${ }^{2}$ Alternatively $\mathrm{W}$ stands for Weyl, as it will be justified later.
} 
supergravity theories with extended local supersymmetry [14-17]. Here we will construct new $\mathrm{W}$-supergravities with $\mathcal{N}=3,4,5,6,7$, namely we will generalize the double copy construction by considering tensor products with one factor being the $\mathcal{N}=3$ supersymmetric gauge theory described above. In particular the $\mathcal{N}=7 \mathrm{~W}$-supergravity will be constructed as a double copy of a non-perturbative theory with a $\mathcal{N}=4$ super Yang-Mills theory:

$$
\mathcal{N}=7 \mathrm{~W}-\text { Sugra } \equiv(\mathcal{N}=4 \mathrm{YM}) \otimes(\mathcal{N}=3 \mathrm{YM})
$$

The two factors respectively refer to the spin-two massive supermultiplets of $\mathcal{N}=4,3$. They are also denoted as Weyl multiplets, since they corresponds to the multiplets of the gauge fields of corresponding local superconformal algebra. As we will discuss, the double copy will contain as lightest multiplet a massive spin-four supermultiplet of the local $\mathcal{N}=7$ supersymmetry algebra, which arises as the tensor product of two spin-two Weyl multiplets [18-24] of the $\mathcal{N}=3$ and $\mathcal{N}=4$ theories.

The double copy construction of gravity theories can be also traced back to the KLTrelations [25] in string theory, as well as from intriguing relations between open and closed string amplitudes [26, 27]. In fact, in the second part of the paper we propose a closely related construction of new, non-perturbative S-folds in string theory, which we will call $\mathrm{W}$-superstrings. In fact, as it is well-known from the "old days" of perturbative string constructions, space-time supersymmetry and world-sheet supersymmetry are closely linked together in string theory. On the heterotic side, four-dimensional perturbative heterotic string constructions lead to $\mathcal{N}$-extended supergravity theories with following number of supercharges:

$$
\mathcal{N}_{H}=1, \quad \mathcal{N}_{H}=2, \quad \text { and } \quad \mathcal{N}_{H}=4,
$$

Here the space-time supercharges originate from the superconformal, right-moving fermionic string sector of the heterotic string. The bosonic, left-moving sector of the heterotic string however does not provide any further space-time supercharges.

In type II constructions, the possible space-time supercharges are obtained by building the tensor product of superconformal left- and right-moving fermionic strings. This leads to several four-dimensional $\mathcal{N}$-extended supergravity theories from perturbative type II string constructions, which are classified as follows:

$$
\begin{array}{lll}
\mathcal{N}_{I I}=1=0_{L}+1_{R}, & \mathcal{N}_{I I}=2=0_{L}+2_{R}, & \mathcal{N}_{I I}=2^{\prime}=1_{L}+1_{R}, \\
\mathcal{N}_{I I}=3=1_{L}+2_{R}, & \mathcal{N}_{I I}=4=0_{L}+4_{R}, & \mathcal{N}_{I I}=4^{\prime}=2_{L}+2_{R}, \\
\mathcal{N}_{I I}=5=1_{L}+4_{R}, & \mathcal{N}_{I I}=6=2_{L}+4_{R}, & \mathcal{N}_{I I}=8=4_{L}+4_{R} .
\end{array}
$$

Explicit constructions of the vertex operators for the type II space-time supercharges as products of left- and right-moving pieces and the associated action of the R-symmetries for all these theories were provided in [28, 29].

It is clear from eq. (1.4) that on the heterotic side it is perturbatively not possible to construct a $\mathcal{N}_{H}=3$ supergravity theory. This is a reflection of the fact that there is also no perturbative $\mathcal{N}=3$ supersymmetric gauge theory. However among the possible type 
II cases in eq. (1.5), there exists a four-dimensional, perturbative $\mathcal{N}_{I I}=3$ supergravity theory. Explicit left-right asymmetric type II constructions in particular with $\mathcal{N}_{I I}=3,5,6$ were provided in [30]. But we also recognize in eq. (1.5) that there is no possible $\mathcal{N}_{I I}=7$ supergravity theory (i.e. with 28 supercharges) from a perturbative type II constructions. Therefore there does not exist a standard $\mathcal{N}=7$ superstring theory with a massless spintwo supermultiplet.

In second part of the paper, we will construct particular string theory quotients, called string theory S-folds, of four-dimensional heterotic and type II strings by dividing with particular elements of the T- and the S-duality groups. Namely, when constructing the string theory S-fold, we will realize that the R-symmetry in field theory is given in terms of certain T-duality transformations. They act as asymmetric rotations on the internal worldsheet coordinates. It follows that the internal six-dimensional space of the four-dimensional string theory S-folds is a non-geometric space, basically given in terms of a particular asymmetric orbifold (see e.g. [31, 32]). Related type string models in two dimensions with 28 chiral supercharges were recently constructed in [6]. In addition we propose a method how the field theory S-duality is implemented into the string construction. The existence of S-duality in four dimensional heterotic string compactifications was first proposed in [33] and further evidence for this conjecture was subsequently given in [34,35]. As we will discuss, also S-duality will act as asymmetric rotation on the uncompactified coordinates in a particular way.

Then, following the same strategy as in field theory, we show that S-folds of heterotic string compactifications lead to heterotic string theories with $\mathcal{N}_{H}=3$ supersymmetry:

$$
\text { String theory } \mathrm{S}-\text { fold : }\left(\mathcal{N}=3_{H}\right) \equiv\left(\mathcal{N}=4_{H}\right) /(T \times S) \text {. }
$$

Moreover, by building the tensor product of a left-moving fermionic string with $\mathcal{N}_{L}=4$ together with an right moving fermionic S-fold with $\mathcal{N}_{R}=3$ we will obtain a type II S-fold with $\mathcal{N}_{I I}=7$ supersymmetry:

$$
\text { String theory } \mathrm{S}-\text { fold : }\left(\mathcal{N}=7_{I I}\right) \equiv(\mathcal{N}=4)_{L} \otimes(\mathcal{N}=3)_{R} .
$$

This $\mathcal{N}=7 \mathrm{~W}$-superstring theory is an entirely higher spin theory without a standard massless spin-2 supermultiplet, and hence it does not possess a standard Langrangian description in terms of a conventional Einstein action coupled to massless spin $=3 / 2$ gravitinos. It is natural to conjecture thats its effective description of the lowest massive states is just $\mathcal{N}=7 \mathrm{~W}$-supergravity, described above.

\section{Double copy construction of massive $\mathrm{W}$-supergravities}

\subsection{Double copy constructions}

In the following we will outline the double copy construction by taking the product of two four-dimensional supersymmetric field theories $\operatorname{QFT}\left(\mathcal{N}_{L}\right)$ and $\operatorname{QFT}\left(\mathcal{N}_{R}\right)$ with $\mathcal{N}_{L}$ resp. with $\mathcal{N}_{L}$ rigid supersymmetry. This leads to four-dimensional supergravity theories with $\mathcal{N}_{L}+\mathcal{N}_{R}$ extended, local supersymmetry:

$$
\operatorname{QFT}\left(\mathcal{N}_{L}\right) \otimes \operatorname{QFT}\left(\mathcal{N}_{R}\right)=\operatorname{Sugra}\left(\mathcal{N}_{L}+\mathcal{N}_{R}\right) .
$$


In order to obtain the spectrum of the double copy, one first has to determine the operators $\Phi_{L}$ and $\Phi_{R}$ with lowest scaling dimension $h_{L}$ and $h_{R}$ in each of the two products. Depending on their scaling dimensions, they correspond either to certain massless or massive superfields with masses $m_{L}$ and $m_{R}$. In order to get the lowest fields in the double copy, we build the tensor product field

$$
\Phi_{L+R}=\Phi_{L} \otimes \Phi_{R}
$$

where one has to demand that $h_{L}=h_{R}$, i.e. $m_{L}=m_{R}$. This condition corresponds to the level matching constraint in string theory.

\subsubsection{Standard massless supergravities as double copy constructions}

First, we can list the nine familiar examples of double copy constructions providing standard extended supergravity theories with massless spin-two supermultiplets [36, 37]. In all these examples the lowest operators of $\operatorname{QFT}\left(\mathcal{N}_{L}\right)$ and $\operatorname{QFT}\left(\mathcal{N}_{R}\right)$ are the spin-one vector multiplets $V$, which correspond to the massless $\mathrm{U}(1)$ super multiplets in each of the two field theories. The double copy is provided by the tensor product of two massless vector multiplets:

$$
\text { Supergravity : } \quad \text { Massless } \operatorname{Spin}(2)=V_{L, \mathcal{N}_{L}} \otimes V_{R, \mathcal{N}_{R}} \text {. }
$$

The massless spin-two supergravity multiplet is always present in the tensor product.

(i) $\mathcal{N}_{L}=\mathcal{N}_{R}=4$. This leads to the well-known extendend $\mathcal{N}=8$ supergravity in the double copy. The lowest operators in each factor are the massless spin-1 vector multiplets (singleton of $\mathcal{N}=4$ ) with 8 bosonic $(\mathrm{B})+8$ fermionic $(\mathrm{F})$ massless states. Their tensor product is the massless spin-2 gravity multiplet of $\mathcal{N}=8$ with $n_{B}=$ $n_{F}=128$ massless states.

(ii) $\mathcal{N}_{L}=2, \mathcal{N}_{R}=4$. This leads to standard massless pure $\mathcal{N}=6$ supergravity.

(iii) $\mathcal{N}_{L}=1, \mathcal{N}_{R}=4$. This leads to standard massless pure $\mathcal{N}=5$ supergravity.

(iv) $\mathcal{N}_{L}=0, \mathcal{N}_{R}=4$. This leads to standard massless pure $\mathcal{N}=4$ supergravity.

(v) $\mathcal{N}_{L}=\mathcal{N}_{R}=2$. This leads to massless $\mathcal{N}=4$ supergravity with two additional massless $\mathcal{N}=4$ vector multiplets.

(vi) $\mathcal{N}_{L}=1, \mathcal{N}_{R}=2$. This leads to massless $\mathcal{N}=3$ supergravity with one additional massless $\mathcal{N}=3$ vector multiplet.

(vii) $\mathcal{N}_{L}=0, \mathcal{N}_{R}=2$. This leads to massless $\mathcal{N}=2$ supergravity with one additional massless $\mathcal{N}=2$ vector multiplet.

(viii) $\mathcal{N}_{L}=\mathcal{N}_{R}=1$. This leads to massless $\mathcal{N}=2$ supergravity with one additional massless $\mathcal{N}=2$ hyper multiplet.

(ix) $\mathcal{N}_{L}=0, \mathcal{N}_{R}=1$. This leads to massless $\mathcal{N}=1$ supergravity with one additional massless $\mathcal{N}=1$ chiral multiplet. 
Note that comparing with the corresponding string constructions, heterotic models can only be obtained if $\mathcal{N}_{L}=0$. Furthermore the only way to obtain massless $\mathcal{N}=3$ supergravity with the double copy method is as in (vi), namely $\mathcal{N}_{L}=1, \mathcal{N}_{R}=2$. This model was obtained as a type II string construction in [30] when it was observed that the three complex scalars parametrize the $C P_{3}$ manifold $\mathrm{SU}(1,3) /(\mathrm{U}(1) \times \mathrm{SU}(3))$ in contrast to the $\mathcal{N}=4$ case where the six real scalars would parametrize $O(1,6) / \mathrm{SO}(6)$. Since the vector $\otimes$ vector tensor product always produces two scalars, it is more difficult to construct pure $\mathcal{N}=1,2,3$ supergravities as double copies. ${ }^{3}$ Viceversa pure supergravities exist for $\mathcal{N} \geq 4$ with $\mathcal{N}_{L}=k, \mathcal{N}_{R}=4, k=0,1,2,4$. The numbers of states of $\mathcal{N}=k+4$ supergravity is $16 \times 2^{k+1}$ for CPT not self conjugate theories $(k=0,1,2)$ and $16 \times 16$ for the $\mathcal{N}=8(k=4) \mathrm{CPT}$ self conjugate one.

\section{$2.24 \mathrm{D}$ massive supermultiplets of $\mathcal{N}$-extended supersymmetry:}

Before we proceed to consider and to construct explicit examples of $\mathrm{W}$-supergravity theories, let us provide a general overview about the structure of massive supermultiplets and tensor products between them. ${ }^{4}$ General massive multiplets of $\mathcal{N}$-extended $4 \mathrm{D}$ supersymmetry [38] with top spin $j_{\max }=j+\frac{\mathcal{N}}{2}$ are obtained by tensoring the smallest multiplet with top spin $\mathcal{N} / 2$ with a spin-j representation of $\mathrm{SU}(2)$. The number of states is then

$$
n_{B}+n_{F}=2^{2 \mathcal{N}}(2 j+1), \quad\left(n_{B}=n_{F}\right) .
$$

For $j=0$ the state of $\operatorname{spin}\left(\frac{\mathcal{N}}{2}-\frac{k}{2}\right)$ is the $\mathrm{k}$-fold antisymmetric (traceless) irreducible representation of $\operatorname{USp}(2 \mathcal{N})$.

If we tensor two massive states of $\mathcal{N}_{1}$ and $\mathcal{N}_{2}$ extended supersymmetry, we get a massive multiplet of $\mathcal{N}_{1}+\mathcal{N}_{2}$ extended supersymmetry with total multiplicity

$$
S=n_{B}+n_{F}=2^{2\left(\mathcal{N}_{1}+\mathcal{N}_{2}\right)}\left(2 j_{1}+1\right)\left(2 j_{2}+1\right), \quad\left(n_{B}=n_{F}\right) .
$$

This representation is reducible into

$$
S=\sum_{j=\left|j_{1}-j_{2}\right|}^{j_{1}+j_{2}}(2 j+1) 2^{2\left(\mathcal{N}_{1}+\mathcal{N}_{2}\right)}
$$

representations. So the representation is irreducible only if $j_{2}=0$ (or $j_{1}=0$ ) for which $S=2^{2\left(\mathcal{N}_{1}+\mathcal{N}_{2}\right)}(2 j+1)$.

In our context the four-dimensional $\mathcal{N}$-extended Weyl multiplets [18] will be of particular importance. They correspond to $4 \mathrm{D}$ massive spin-two fields of the of $\mathcal{N}$-extended supersymmetry. The generic spin-two massive multiplet (Weyl multiplet) is obtained by tensoring the smallest massive representation of $\mathcal{N}$-extended supersymmetry (with $j_{\max }=\frac{\mathcal{N}}{2}$ ) with spin $j=2-\frac{\mathcal{N}}{2}$. The number of states of such spin-two multiplet are therefore $n_{B}+n_{F}=(5-\mathcal{N}) 2^{2 \mathcal{N}}$. In particular for $\mathcal{N}=4$ it is the smallest long massive multiplet.

\footnotetext{
${ }^{3} \mathrm{~A}$ way to construct these theories was demonstrated in $[42,43]$.

${ }^{4}$ For massive representations we mean long multiplets without central charges.
} 
So let us list all the possible Weyl supermultiplets, denoted by $W_{\mathcal{N}=k}(k=1,2,3,4)$, of supersymmetric field theories. The spin-two massive multiplet of $\mathcal{N}=4$ is irreducible with $n_{B}+n_{F}=2^{8}=256$ with states in $\operatorname{USp}(8)$ representations [19]:

$$
W_{\mathcal{N}=4}: \operatorname{Spin}(2)+\underline{8} \times \operatorname{Spin}(3 / 2)+\underline{27} \times \operatorname{Spin}(1)+\underline{48} \times \operatorname{Spin}(1 / 2)+\underline{42} \times \operatorname{Spin}(0) .
$$

The spin-two massive Weyl multiplet of $\mathcal{N}=3$ is obtained by tensoring the smallest $j_{\max }=\frac{\mathcal{N}}{2}=\frac{3}{2}$ massive multiplet with $j=\frac{1}{2}$, and it is irreducible with states in $\operatorname{USp}(6)$ representations where $n_{B}+n_{F}=2 \times 2^{6}=128$ [20]:

$$
\begin{aligned}
& W_{\mathcal{N}=3}: \quad \operatorname{Spin}(2)+\underline{6} \times \operatorname{Spin}(3 / 2)+(\underline{14}+\underline{1}) \times \operatorname{Spin}(1) \\
&+\left(\underline{14^{\prime}}+\underline{6}\right) \times \operatorname{Spin}(1 / 2)+\underline{14} \times \operatorname{Spin}(0) .
\end{aligned}
$$

Finally for $\mathcal{N}=2,1$ one obtains $[21-23]$

$$
W_{\mathcal{N}=2}: \operatorname{Spin}(2)+\underline{4} \times \operatorname{Spin}(3 / 2)+(\underline{5}+\underline{1}) \times \operatorname{Spin}(1)+\underline{4} \times \operatorname{Spin}(1 / 2)+\operatorname{Spin}(0),
$$

where $n_{B}+n_{F}=48$.

$$
W_{\mathcal{N}=1}: \operatorname{Spin}(2)+\underline{2} \times \operatorname{Spin}(3 / 2)+\operatorname{Spin}(1) .
$$

with $n_{B}+n_{F}=16$ states.

The field theory realization of Weyl multiplets allows one to write a unique Lagrangian for all conformal supergravities $(\mathcal{N} \leq 4)[39-41,44,45]$. In view of the fact that massive spin-two can also be seen as Weyl multiplets, it is instructive to indicate their $\mathrm{U}(\mathcal{N})$ Rsymmetry quantum numbers. This identification is achieved by decomposing $\operatorname{USp}(2 \mathcal{N})$ into $\mathrm{U}(\mathcal{N})$ representations. The states, which are not gauge fields, are auxiliary fields of extended superconformal supergravity. For example in the $\mathcal{N}=1$ spin-two multiplet $(2,2(3 / 2), 1)$ there are the Weyl graviton, the R-symmetry $\mathrm{U}(1)$ gauge bosons and two spin$3 / 2$ gauge fields of $Q$ and $S$ supersymmetry. There are no auxiliary fields in this case. For the higher cases there are always $2 \mathcal{N}$ gravitini for $Q$ and $S$ supersymmetry. The $\mathrm{U}(\mathcal{N})$ gauge fields must be in the spin-one sector of the massive multiplet. For $\mathcal{N}=2,3,4$ the spin-one representations are easily seen to always contain the adjoint representation of $\mathrm{U}(\mathcal{N})$. For example in $\mathcal{N}=3$ the spin-one decomposes into $(\underline{14}+\underline{1}) \rightarrow \underline{8}+\underline{3}+\underline{\overline{3}}+\underline{1}$ under the $\mathrm{U}(3)$, where the $\underline{8}+\underline{1}$ are the gauge bosons the $\mathrm{U}(3)$ R-symmetry. In $\mathcal{N}=4$ the $\underline{27}$ of $\mathrm{USp}(8)$ decomposes into $\underline{15}+\underline{6}+\underline{\overline{6}}$ of $\mathrm{SU}(4)$. Here the $\underline{15}$ correspond to the gauge bosons of the SU(4) R-symmetry, since there is no $\mathrm{U}(1)$ factor in the gauge symmetry. The other states correspond to auxiliary fields, whose presence have the double role of completing the spin-two massive multiplet or, in the field theory side, to ensure the same number of bosonic and fermionic off-shell degrees of freedom in the Weyl multiplet.

\section{$2.3 \quad$ The $\mathcal{N}=3$ S-fold}

Let us recall how, starting from the $\mathcal{N}=4$ supersymmetric SYM theories, how the strongly coupled $\mathcal{N}=3$ supersymmetric field theory is obtained by a projection, which is a combination of a group element of the R-symmetry and the $\mathrm{SL}(2, \mathbb{Z})$ S-duality automorphism group [7]. The 16 supercharges of four-dimensional $\mathcal{N}=4$ supersymmetry 
are denoted as $Q^{\alpha, A}$ and $Q^{\dot{\alpha}, \dot{A}}$. Here $\alpha=1,2$ and $\dot{\alpha}=1,2$ are the spinor indices of the $(\underline{2}, \underline{1})+(\underline{1}, \underline{2})=\underline{2}_{S}+\underline{2}_{c}$ dimensional spinor representations of the four-dimensional Lorentz group $\mathrm{SO}(1,3) \equiv \mathrm{SL}(2) \times \mathrm{SL}(2)$. Furthermore the indices $A=1, \ldots, 4$ and $\dot{A}=1, \ldots, 4$ denote the spinor and anti-spinor indices of the two inequivalent spinor representations $\underline{4}_{s}$ and $\underline{4}_{c}$ of the four-dimensional R-symmetry group $\mathrm{SO}(6)$. More details on the transformation rules of the supercharges are given in the appendix.

Now following [7], in order to break $\mathcal{N}=4$ supersymmetry down to $\mathcal{N}=3$ supersymmetry, we will choose a particular $\mathbb{Z}_{4}$ rotation, which is embedded in the $\mathrm{SO}(6)_{R}$ R-symmetry group and which acts on the $\mathcal{N}=4$ supercharges as follows:

$$
\begin{array}{ll}
\mathrm{R}-\text { symmetry }: & Q^{\alpha, A} \rightarrow e^{-\frac{i \pi\left(w_{1}^{A}+w_{2}^{A}+w_{3}^{A}\right)}{2}} Q^{\alpha, A}, \\
& Q^{\dot{\alpha}, \dot{A}} \rightarrow e^{-\frac{i \pi\left(w_{1}^{\dot{A}}+w_{2}^{\dot{A}}+w_{3}^{\dot{A}}\right)}{2}} Q^{\dot{\alpha}, \dot{A}},
\end{array}
$$

where the $\left(w_{1}^{A}, w_{2}^{A}, w_{3}^{A}\right)=\left( \pm \frac{1}{2}, \pm \frac{1}{2}, \pm \frac{1}{2}\right)$ (even number of - signs) are spinor weights of $\underline{4}_{s}$ of the $\mathrm{SO}(6)_{R}$ group, whereas the $\left(w_{1}^{\dot{A}}, w_{2}^{\dot{A}}, w_{3}^{\dot{A}}\right)=\left( \pm \frac{1}{2}, \pm \frac{1}{2}, \pm \frac{1}{2}\right)$ (odd number of - signs) are spinor weights of $\underline{4}_{c}$ representation. The supercharges specifically transform under the $\mathbb{Z}_{4}$ R-symmetry rotation as:

$$
\begin{aligned}
Q^{\alpha, A}: \quad & \left(\frac{1}{2}, \frac{1}{2}, \frac{1}{2}\right) \rightarrow e^{-\frac{3 i \pi}{4}}\left(\frac{1}{2}, \frac{1}{2}, \frac{1}{2}\right), \\
& \left(-\frac{1}{2},-\frac{1}{2}, \frac{1}{2}\right) \rightarrow e^{\frac{i \pi}{4}}\left(-\frac{1}{2},-\frac{1}{2}, \frac{1}{2}\right), \\
& \left(-\frac{1}{2}, \frac{1}{2},-\frac{1}{2}\right) \rightarrow e^{\frac{i \pi}{4}}\left(-\frac{1}{2}, \frac{1}{2},-\frac{1}{2}\right), \\
& \left(\frac{1}{2},-\frac{1}{2},-\frac{1}{2}\right) \rightarrow e^{\frac{i \pi}{4}}\left(\frac{1}{2},-\frac{1}{2},-\frac{1}{2}\right), \\
Q^{\dot{\alpha}, \dot{A}}: \quad & \left(-\frac{1}{2},-\frac{1}{2},-\frac{1}{2}\right) \rightarrow e^{\frac{3 i \pi}{4}}\left(-\frac{1}{2},-\frac{1}{2},-\frac{1}{2}\right), \\
& \left(-\frac{1}{2}, \frac{1}{2}, \frac{1}{2}\right) \rightarrow e^{-\frac{i \pi}{4}}\left(-\frac{1}{2}, \frac{1}{2}, \frac{1}{2}\right), \\
& \left(\frac{1}{2},-\frac{1}{2}, \frac{1}{2}\right) \rightarrow e^{-\frac{i \pi}{4}}\left(\frac{1}{2},-\frac{1}{2}, \frac{1}{2}\right), \\
& \left(\frac{1}{2}, \frac{1}{2},-\frac{1}{2}\right) \rightarrow e^{\frac{-i \pi}{4}}\left(\frac{1}{2}, \frac{1}{2},-\frac{1}{2}\right) .
\end{aligned}
$$

Next we will consider the S-duality transformations. The S-duality is a nonperturbative symmetry of $\mathcal{N}=4$ supersymmetric SYM theories. As in [7] we will choose a particular order four element of the $\mathrm{SL}(2, \mathbb{Z})$ S-duality group, which acts on all eight supercharges $Q^{\alpha A}$ in the same way:

$$
\mathrm{S}-\text { duality }: \quad Q^{\alpha, A} \rightarrow e^{-\frac{i \pi}{4}} Q^{\alpha, A} .
$$

On the remaining eight supercharges $Q^{\dot{\alpha}, \dot{A}}$ S-duality acts in the opposite way:

$$
\text { S-duality : } \quad Q^{\dot{\alpha} \dot{A}} \rightarrow e^{\frac{i \pi}{4}} Q^{\dot{\alpha}, \dot{A}} .
$$


Combining these transformation rules with the R-symmetry transformations in eq. (2.12), it immediately follows that by projection on the invariant charges the combined action of $R \cdot S$, called S-fold projection, leaves twelve supercharges invariant, i.e. leads to $\mathcal{N}=3$ supersymmetry. As we will discuss later, the implementation of the S-duality transformations in the heterotic string will be quite subtle.

Now let us determine the additional operators, which are invariant under the S-fold projection, of the non-perturbative $\mathcal{N}=3$ field theory. This theory is strongly coupled and there is no Langragian description of this theory. ${ }^{5}$ The crucial point is that in super YangMills, the S-fold projection eliminates the massless fields altogether. According to [10], the singleton of the $\mathcal{N}=3$ field theory is described by a superfield strength and an $\mathrm{SU}(3)$ triplet $V_{i}(x, \theta)$, which decomposes under $\mathcal{N}=1$ into a vector multiplet and three chiral multiplets. However the singleton of scaling dimension $h=1$ is itself not invariant under the S-fold projection. The invariant $\mathcal{N}=3$ operators possess scaling dimension $h=2 p$ and have the following form:

$$
\Phi^{2 p}=\operatorname{Tr}\left(V_{i 1} \bar{V}_{i 2} \ldots V_{i 2 p-1} \bar{V}_{i 2 p}\right) .
$$

The lowest operator, denoted as $W_{\mathcal{N}=3} \equiv \Phi^{2}$, has scaling dimension $h=2$ and specifically is given by

$$
W_{\mathcal{N}=3}=\operatorname{Tr}\left(V_{i} \bar{V}^{j}-\frac{1}{3} \delta_{i}^{j} V_{k} \bar{V}^{k}\right) .
$$

This operator is nothing else than the energy momentum tensor (i.e. supercurrent) of the $\mathcal{N}=3$ field theory. It corresponds to the massive $\mathcal{N}=3$ spin-two super-Weyl multiplet with, as already shown before, the following decomposition of massive component fields:

$$
\begin{aligned}
W_{\mathcal{N}=3}: \quad \operatorname{Spin}(2)+\underline{6} & \times \operatorname{Spin}(3 / 2)+(\underline{14}+\underline{1}) \times \operatorname{Spin}(1) \\
& +\left(\underline{14^{\prime}}+\underline{6}\right) \times \operatorname{Spin}(1 / 2)+\underline{14^{\prime}} \times \operatorname{Spin}(0) .
\end{aligned}
$$

It contains $n_{B}=n_{F}=64$ degrees of freedom. These massive states were also anticipated before in [24]. The multiplicities in front of the different fields denote the representations with respect to the group $\operatorname{USp}(6)$, which is the relevant automorphism group of the massive $\mathcal{N}=3$ super algebra. Note that the $\mathcal{N}=3$ massive spin-two multiplet is not the same as the $\mathcal{N}=4$ massive spin-two multiplet.

In addition, one can also consider the $h=2$ operator

$$
w_{\mathcal{N}=3}=\operatorname{Tr} V_{i} V_{j}
$$

This operator correspond to a massive spin $=3 / 2$ supermultiplet of $\mathcal{N}=3$ with the following field content:

$$
w_{\mathcal{N}=3}: \quad \operatorname{Spin}(3 / 2)+\underline{6} \times \operatorname{Spin}(1)+\underline{14} \times \operatorname{Spin}(1 / 2)+\underline{14} \times \operatorname{Spin}(0) .
$$

\footnotetext{
${ }^{5}$ If $\mathrm{SU}(2,2 / 3)$ is the boundary superconformal algebra, then the dual bulk theory should be $\mathcal{N}=6 A d S_{5}$ supergravity which is not the same as $\mathcal{N}=8 A d S_{5}$ supergravity [10]. The same projection, which involves an S-duality transformation leading from $\mathcal{N}=4$ to $\mathcal{N}=3$ supersymmetry on the boundary, reduces in the bulk $\mathcal{N}=8$ to $\mathcal{N}=6$ supergravity.
} 
However this operator, which contains $n_{B}=n_{F}=32$ fields, is not invariant under the $R \cdot S$ projection.

The operators $W_{\mathcal{N}=3}$ and $w_{\mathcal{N}=3}$ immediately follow from the decomposition of the $\mathcal{N}=4$ supercurrent $W_{\mathcal{N}=4}$. As already shown, this current contains $n_{B}=n_{F}=128$ component fields:

$$
W_{\mathcal{N}=4}: \quad \operatorname{Spin}(2)+\underline{8} \times \operatorname{Spin}(3 / 2)+\underline{27} \times \operatorname{Spin}(1)+\underline{48} \times \operatorname{Spin}(1 / 2)+\underline{42} \times \operatorname{Spin}(0),
$$

with multiplicities with respect to the massive $\mathcal{N}=4$ automorphism group $\operatorname{USp}(8) . W_{\mathcal{N}=4}$ decomposes under the $\mathcal{N}=3$ currents as: ${ }^{6}$

$$
W_{\mathcal{N}=4}=W_{\mathcal{N}=3}+2 \times w_{\mathcal{N}=3} .
$$

As said before, only $W_{\mathcal{N}=3}$ survives the S-fold projection.

\subsection{Non-standard massive $\mathrm{W}$-supergravities as double copy constructions}

So far we did not consider $\mathcal{N}=3$ rigid field theory in one of the factors, say in $\operatorname{QFT}\left(\mathcal{N}_{R}\right)$, of the double copy construction. The W-supergravities always have at least one $\operatorname{QFT}(\mathcal{N}=3)$ field theory in one of the factors of the double construction:

$$
\mathrm{W}-\text { supergravity }=\operatorname{QFT}\left(\mathcal{N}_{L}\right) \otimes \operatorname{QFT}\left(\mathcal{N}_{R}=3\right)
$$

As we discussed in the last section, in $\operatorname{QFT}(\mathcal{N}=3)$ the lowest operator (which is invariant under the S-fold twist) is the supercurrent (energy momentum tensor) of the $\mathcal{N}=3$ field theory. Strictly speaking the supercurrent (being the system superconformal) has the same structure as the super-Weyl multiplet, which contains the spin-two and the other gauge fields of the superconformal algebra. Therefore, in the tensor product, the lowest field appearing is the $\mathcal{N}=3$ massive spin-two Weyl multiplet with $n_{B}=n_{F}=64$ massive states. Due to the constraint $h_{L}=h_{R}$, the Weyl multiplet of $\operatorname{QFT}\left(\mathcal{N}_{R}=3\right) \operatorname{must}$ be tensored also with massive fields of the same mass, namely also with the spin-two massive Weyl supermultiplet from $\mathrm{QFT}\left(\mathcal{N}_{L}\right)$. This leads to a massive spin-four field $\Phi_{L+R, \mathcal{N}_{L}+3}^{4}$ as lowest possible operator in massive $\mathrm{W}$-supergravity:

$$
\mathrm{W} \text { - supergravity : } \quad \Phi_{L+R, \mathcal{N}_{L}+3}^{4}=W_{L, \mathcal{N}_{L}} \otimes W_{R, \mathcal{N}_{R}=3} .
$$

Knowing this we can now proceed and give an overview over the following five nonstandard, massive $\mathrm{W}$-supergravity theories:

(i) $\mathcal{N}_{L}=4, \mathcal{N}_{R}=3$. This leads to massive $\mathcal{N}=7 \mathrm{~W}$-supergravity. Due to the level matching constraint, we have to take the massive spin-2 multiplet of $\mathcal{N}=4$ in the tensor product, which contains $n_{B}=n_{F}=128$ massive states. So the relevant tensor product leads to a massive spin- 4 of $\mathcal{N}=7$. It contains $n_{B}=n_{F}=16384$ massive states. This theory and its spectrum will be discussed in more detail in section 2.5.

\footnotetext{
${ }^{6}$ All these multiplets can be regarded as massless multiplets in the five-dimensional, holographically dual $\mathcal{N}=8$ and $\mathcal{N}=6$ supergravity theories.
} 
(ii) $\mathcal{N}_{L}=\mathcal{N}_{R}=3$. This leads to massive $\mathcal{N}=6 \mathrm{~W}$-supergravity.

(iii) $\mathcal{N}_{L}=2, \mathcal{N}_{R}=3$. This leads to massive $\mathcal{N}=5 \mathrm{~W}$-supergravity.

(iv) $\mathcal{N}_{L}=1, \mathcal{N}_{R}=3$. This leads to massive $\mathcal{N}=4 \mathrm{~W}$-supergravity.

(v) $\mathcal{N}_{L}=0, \mathcal{N}_{R}=3$. This leads to massive $\mathcal{N}=3 \mathrm{~W}$-supergravity. It is the only case which can be constructed as a heterotic W-string. Its heterotic spectrum will be discussed in more detail in section 4.4.

All double copy constructions, standard massless supergravity theories as well as massive $\mathrm{W}$-supergravity theories, have in fact the same interpretation, we are always tensoring superconformal field theories, for the spin-one case they are Yang-Mills, for the spin-two case they are conformal (Weyl) gravity. They are in any case local field theories. The result is that for spin-one tensoring we have massless spin-two gravity, while for the spin-two tensoring we have massive spin-four W-supergravity, which therefore exists for $\mathcal{N}=7$.

Finally note that the cases $\mathcal{N}_{L}+\mathcal{N}_{R}=3,4,5,6$ can be also realized as standard supergravities with massless spin-two. So for these cases there exist two inequivalent double copies: standard spin-two supergravity and massive spin-four W-supergravity. Only for $\mathcal{N}=7$ there exist only the massive spin-four double copy W-supergravity construction.

\subsection{Massive $\mathcal{N}=7 \mathrm{~W}$-supergravity}

In this section we focus on the construction of the $\mathcal{N}=7 \mathrm{~W}$-supergravity theory. It is constructed as a tensor product of $(\mathcal{N}=4) \times(\mathcal{N}=3)$ field theories. Hence the first, massive higher spin field in the $\mathcal{N}=7 \mathrm{~W}$-supergravity theory is obtained from the product $W_{L, \mathcal{N}_{L}=4} \otimes W_{R, \mathcal{N}_{R}=3} \simeq(\text { Spin }=2)_{\mathcal{N}=4} \otimes(\text { Spin }=2)_{\mathcal{N}=3}$. This tensor product starts with one spin-four field and has the following decomposition:

$$
\begin{gathered}
\left(\operatorname{Spin}(2)+8 \times \operatorname{Spin}\left(\frac{3}{2}\right)+27 \times \operatorname{Spin}(1)+48 \times \operatorname{Spin}\left(\frac{1}{2}\right)+42 \times \operatorname{Spin}(0)\right)_{\mathcal{N}=4} \\
\otimes\left(\operatorname{Spin}(2)+6 \times \operatorname{Spin}\left(\frac{3}{2}\right)+15 \times \operatorname{Spin}(1)+20 \times \operatorname{Spin}\left(\frac{1}{2}\right)+14 \times \operatorname{Spin}(0)\right)_{\mathcal{N}=3} \\
=\left(\operatorname{Spin}(4)+\underline{14}_{1} \times \operatorname{Spin}\left(\frac{7}{2}\right)+\left(\underline{90}_{2}+\underline{1}\right) \times \operatorname{Spin}(3)+\left(\underline{350}_{3}+\underline{14}_{1}\right) \times \operatorname{Spin}\left(\frac{5}{2}\right)\right. \\
+\left(\underline{90}_{2}+\underline{910}_{4}\right) \times \operatorname{Spin}(2)+\left(\underline{350}_{3}+\underline{1638}_{5}\right) \times \operatorname{Spin}\left(\frac{3}{2}\right)+\left(\underline{2002}_{6}+\underline{910}_{4}\right) \times \operatorname{Spin}(1) \\
\left.+\left(\underline{1430}_{5}+\underline{1638}_{5}\right) \times \operatorname{Spin}\left(\frac{1}{2}\right)+\underline{2002}_{6} \times \operatorname{Spin}(0)\right)_{\mathcal{N}=7},
\end{gathered}
$$

where the two factors contain $2^{8}$ and $2^{7}$ states, respectively. The multiplicities of these massive states fall into representations of the group USp(14), which is the automorphism group of $\mathcal{N}=7$ supersymmetry for massive states, where the label means an irreducible, traceless, n-fold USp(14) antisymmetric representation. It contains $n_{B}+n_{F}=2^{15}=32768$ degrees of freedom. Note that this massive multiplet does not constitute a proper $\mathcal{N}=8$ supermultiplet, but it only transforms under $\mathcal{N}=7$ supersymmetry transformations. It 
contains 1000 massive graviton-like, spin-two fields. Since it arises from the tensor product of a strongly coupled $\mathcal{N}=3 \mathrm{SYM}$ theory, it is conceivable that this spin-four multiplet also only exists in a strongly coupled theory without a Langrangian description. As we will show in the next section, the $\mathcal{N}=7$ spin-four multiplet arises at the first mass level in the corresponding superstring S-fold construction. Furthermore note that the $\mathcal{N}=7 \mathrm{~W}$ supergravity theory can be also obtained as S-fold from $\mathcal{N}=8$ supergravity after including an $\mathcal{N}=8$ spin-four multiplet in a suitable way.

\section{String S-fold construction - Implementation of R-symmetry and S- duality transformations in heterotic and type II string theory}

Now we want to construct analogous S-folds in type II and heterotic string theories. We call these theories W-strings, since they do not contain any massless excitations, but only massive string excitations of higher spin. Their effective description is then supposedly given in terms of the massive $\mathrm{W}$-supergravity theories, discussed before.

Recall that the S-fold projection in field theory include a discrete R-symmetry transformations times a discrete S-duality transformations. As we will now see, the field theory R-symmetry transformations will be realized as special kind of T-duality transformations in string theory. In fact, discrete elements of the T-duality group act like discrete Rsymmetry transformations, which are subgroups of the $\mathrm{SO}(6)_{R}$ automorphism group of the $\mathcal{N}=4$ supersymmetry algebra, as it was discussed in [47, 48]. Hence the R-charges of the space-time fields are closely related to the internal modular T-duality transformation.

In the following we will first consider the right-moving world-sheet degrees of freedom of the heterotic or type II string on $T^{6}$. The internal coordinates of the six-torus are denoted by $Y^{I}$, with $I=1, \ldots, 6$. In the four-dimensional uncompactified space-time we go to the light-cone gauge, and the relevant transversal spatial coordinates, corresponding to the transversal little group $\mathrm{SO}(2)_{T}$, are denoted by $X^{i}$ with $i=1,2$. In the 10-dimensional, right-moving sector of the heterotic string we have a world-sheet theory with local $n=1$ world-sheet supersymmetry. The right-moving word-sheet fields of the R-NS fermionic string are then given as:

$$
X^{i}(z), \quad Y^{I}(z), \quad \psi^{i}(z), \quad \lambda^{I}(z), \quad \phi(z) .
$$

The $\psi^{i}(z)$ and the $\lambda^{I}(z)$ are world-fermions and $\phi(z)$ is the bosonized superconformal ghost field. We can introduce complex coordinates on the torus,

$$
Z^{K}(z)=Y^{2 K-1}(z)+i Y^{2 K}(z), \quad K=1, \ldots, 3 .
$$

Furthermore it will be convenient to introduce complex fermions as

$$
\Psi^{0}(z)=\psi^{1}(z)+i \psi^{2}(z), \quad \Psi^{K}(z)=\lambda^{2 K-1}(z)+i \lambda^{2 K}(z) .
$$

Bosonization of these complex world-sheet fermions is then performed in the standard way:

$$
\Psi^{0}(z)=e^{i H^{0}(z)}, \quad \Psi^{K}(z)=e^{i H^{K}(z)}
$$

The $H^{0}(z), H^{K}(z)$ are four chiral (right-moving) bosons on the world-sheet. 
In the left-moving sector of the heterotic string there are the following worldsheet fields:

$$
\bar{X}^{i}(\bar{z}), \quad \bar{Y}^{I}(\bar{z}), \quad \bar{Y}^{I^{\prime}}(\bar{z})
$$

Here the $\bar{X}^{i}(\bar{z})$ the left-moving uncompactified coordinates, the $\bar{Y}^{I}(\bar{z})$ are the left-moving coordinates on $T^{6}$ and the $\bar{Y}^{I^{\prime}}(\bar{z})\left(I^{\prime}=1, \ldots, 16\right)$ are the additional bosonic coordinates that are associated to the $\mathrm{U}(1)^{16}$ Cartan sub-algebra of the additional heterotic gauge group $G_{L}$ of rank 16. In case of the type II sring, the $\bar{Y}^{I^{\prime}}(\bar{z})$ are absent and instead there are also the left-moving fermions on the world-sheet.

The relevant massless bosonic spectrum and their associated vertex operators (in the canonical ghost picture) are given as follows:

$$
\begin{aligned}
\text { graviton : } & G^{(i j)}=\bar{\partial} \bar{X}^{(i}(\bar{z}) \psi^{j)}(z) e^{-\phi(z)}, \\
\text { anti - sym. tensor : } & B^{[i j]}=\bar{\partial} \bar{X}^{[i}(\bar{z}) \psi^{j]}(z) e^{-\phi(z)}, \\
\text { dilaton : } & \Phi=\bar{\partial} \bar{X}^{i}(\bar{z}) \psi^{i}(z) e^{-\phi(z)}, \\
3 \text { complex structure moduli of } \mathrm{T}^{6}: & \tau^{K}=\bar{\partial} Z^{K}(\bar{z}) \Psi^{K}(z) e^{-\phi(z)}, \\
3 \text { Kaehler moduli of } \mathrm{T}^{6}: & \rho^{K}=\bar{\partial} \bar{Z}^{K}(\bar{z}) \Psi^{K}(z) e^{-\phi(z)} .
\end{aligned}
$$

The superpartners of all these states are obtained by performing the operator product of the associated vertex operators with the vertex operators of the four supercharges of the $\mathcal{N}=4$ space-time supersymmetry, where the four space-time supercharges $Q^{\alpha, A}$ are coming from the superconformal, right-moving sector of the heterotic string. The spinors are realized on the world-sheet by the space-time $\mathrm{SO}(2)_{T}$ spin fields $S^{a}(z)$ together with the internal spin fields $S^{A}(z)$ of the $\mathrm{SO}(6)_{R}$ R-symmetry group. In the canonical $-1 / 2$ ghost picture the four supercharges of positive helicity take the following specific form:

$$
\begin{aligned}
Q^{\alpha=\frac{1}{2}, A} & =\oint \frac{d z}{2 i \pi} S^{a=\frac{1}{2}}(z) S^{A}(z) e^{-\frac{\phi(z)+i H_{\star}(z)}{2}} \\
& =\oint \frac{d z}{2 i \pi} e^{\frac{1}{2} i H^{0}(z)} e^{i w_{K}^{A} H^{K}(z)} e^{-\frac{\phi(z)+i H_{\star}(z)}{2}}
\end{aligned}
$$

whereas the four negative helicity supercharges are given as

$$
\begin{aligned}
Q^{\alpha=-\frac{1}{2}, A} & =\oint \frac{d z}{2 i \pi} S^{a=-\frac{1}{2}}(z) S^{A}(z) e^{-\frac{\phi(z)-i H_{\star}(z)}{2}} \\
& =\oint \frac{d z}{2 i \pi} e^{-\frac{1}{2} i H^{0}(z)} e^{i w_{K}^{A} H^{K}(z)} e^{-\frac{\phi(z)-i H_{\star}(z)}{2}}
\end{aligned}
$$

Here the four vectors $w_{K}^{A}=\vec{w}^{A}=\left( \pm \frac{1}{2}, \pm \frac{1}{2}, \pm \frac{1}{2}\right)$ (even number of - signs) are the spinorial weight vectors of the $\underline{4}_{s}$ representation of the internal R-symmetry group $\mathrm{SO}(6)_{R}$. Furthermore, $\phi(z)$ is the bosonised superghost field and $H_{\star}(z)$ is the bosonisation of the longitudinal (lightcone) spacetime directions. Here the index $a=\frac{1}{2}\left(-\frac{1}{2}\right)$ corresponds to (anti)-spinor indices of the transversal little group $\mathrm{SO}(2)_{T}$. Note that the index $a$ is not identical to the spinor index $\alpha$ (or $\dot{\alpha}$ ) of the four-dimensional Lorentz group $\mathrm{SO}(1,3)$, which 
was introduced in the previous section. ${ }^{7}$ Also note that we have adopted in eqs. (3.7) and eqs. (3.8) the convention for the supercharges $Q^{\alpha, A}$ to picking an even number of minus signs in $\vec{w}^{A}$. This implies the transversal spinor index $a$ is correlated with the longitudinal spinor index $b$, as it is indicated in eq. (3.9). For completeness we also list the expressions for the eight conjugate supercharges $Q^{\dot{\alpha}, \dot{A}}$ :

$$
\begin{aligned}
Q^{\dot{\alpha}=\frac{1}{2}, \dot{A}} & =\oint \frac{d z}{2 i \pi} S^{a=\frac{1}{2}}(z) S^{\dot{A}}(z) e^{-\frac{\phi(z)-i H_{\star}(z)}{2}} \\
& =\oint \frac{d z}{2 i \pi} e^{\frac{1}{2} i H^{0}(z)} e^{i w_{K}^{\dot{A}} H^{K}(z)} e^{-\frac{\phi(z)-i H_{\star}(z)}{2}} \\
Q^{\dot{\alpha}=-\frac{1}{2}, \dot{A}} & =\oint \frac{d z}{2 i \pi} S^{a=-\frac{1}{2}}(z) S^{\dot{A}}(z) e^{-\frac{\phi(z)+i H_{\star}(z)}{2}} \\
& =\oint \frac{d z}{2 i \pi} e^{-\frac{1}{2} i H^{0}(z)} e^{i w_{K}^{\dot{A}} H^{K}(z)} e^{-\frac{\phi(z)+i H_{\star}(z)}{2}} .
\end{aligned}
$$

Finally, the type II supercharges from the left-moving sector are constructed in the same way as their right-moving counterparts.

Now we want to implement the R-symmetry transformations as well as the S-duality transformations of $\mathcal{N}=4$ field theory, which we have described in the last section, in the heterotic and type II string. To recall, in field theory the S-fold projection was defined by looking at the discrete R- and S-duality charges of the various fields, where the R-charges originate from the internal symmetries, and the S-duality charges are determined by the helicity degrees of freedom of the fields. Then a proper linear combination of the R- and S-charges defines the S-fold projection, where one keeps only the states invariant under the combined, discrete transformation. In string theory, the same procedure can be performed: all massless and massive states are classified by the same R- and S-charges as in field theory. Therefore one can perform the same truncation of the massless and massive spectrum as in field theory. Here we want to propose an interpretation how this truncation can be regarded as certain rotations on the left- and right-moving world-sheet degrees of freedom of the heterotic and the type II string theories. There will be two essential requirements:

(i) The action on the four right-moving supercharges of the heterotic/type II string is like in field theory.

(ii) There is no action on the four left-moving supercharges of the type II string.

\footnotetext{
${ }^{7}$ The precise relation between $a$ and $\alpha$ or $\dot{\alpha}$ is as follows: decomposing $\mathrm{SO}(1,3)$ as $\mathrm{SO}(1,3) \supset \mathrm{SO}(2)_{T} \times$ $\mathrm{SO}(2)_{L}$, the two dimensional Lorentz spinors with spinor indices $\alpha=\frac{1}{2},-\frac{1}{2}$ have the following charges $(a, b)$ under $\mathrm{SO}(2)_{T} \times \mathrm{SO}(2)_{L}$ :

$$
\alpha=1 / 2: \quad(a, b)=\left(\frac{1}{2}, \frac{1}{2}\right), \quad \alpha=-1 / 2: \quad(a, b)=\left(-\frac{1}{2},-\frac{1}{2}\right),
$$

For the two spinors with spinor indices $\dot{\alpha}$ we get likewise:

$$
\dot{\alpha}=1 / 2: \quad(a, b)=\left(\frac{1}{2},-\frac{1}{2}\right), \quad \dot{\alpha}=-1 / 2: \quad(a, b)=\left(-\frac{1}{2}, \frac{1}{2}\right),
$$


Therefore, from the word-sheet point of view, the string construction has to be completely left-right asymmetric, where the $\mathbb{Z}_{4}$ S-fold projection must only act on the rightmoving string degrees of freedom, but not at all on the left-moving degrees of freedom. As we will now discuss we will satisfy these two requirements in the following way:

- R-symmetry: here we can follow the construction in [6] and also in [32]. The $\mathbb{Z}_{4}$ rotation acts on the internal right-moving six coordinates plus the corresponding world-sheet fermions, but is leaving the left-moving internal coordinates and the leftmoving internal world-sheet fermions invariant. This asymmetric transformation just corresponds to a twist by a T-duality transformations, which means that the internal background can be view as a special kind of non-geometric T-fold space. All massless geometric moduli are projected out by this transformation.

- S-duality: here we will propose that the asymmetric $\mathbb{Z}_{4}$ projection will be realized as a left-right asymmetric $\mathbb{Z}_{4}$ rotation on the two transversal right-moving space-time coordinates plus the corresponding world-sheet fermions. The left-moving space-time coordinates and world-sheet fermions are not rotated. Therefore, although not being compactified, the transversal space coordinates of the closed string are treated in a left- and right-asymmetric way. This is a new and non-standard feature of the string theory S-fold construction, which means that the closed string boundary conditions with respect to $\sigma$ - and $\tau$-translations are chosen with a different $\mathbb{Z}_{4}$-phase factor for the left- and right-moving coordinates (see [46] for a discussion on this issue). The different treatment of the left- and right-moving uncompactified string coordinates can be also motivated in the framework of double field theory. Performing the leftright asymmetric projection basically means that also the uncompactified space is a non-geometric background. As we will see, the projection does not allow anymore for massless states, and in particular there is non massless graviton anymore after the S-fold projection. Therefore the background is completely rigid. Nevertheless all states can be still classified according their helicity quantum numbers and the transversal Lorentz group still acts in the correct way on all states. In this sense, Lorentz invariance will be still preserved. In other words, the charges of all states under the left-right asymmetric rotations agree with the S-duality (helicity) charges of the corresponding fields in field theory, and the projection on the invariant states will be the same as in field theory.

More specifically, in the light-cone gauge the asymmetric $\mathbb{Z}_{4}$ projection includes three simultaneous actions on the physical fields: first it will act on the axion-dilaton S-field in a non-trivial way and, as an effect of it, the S-field is projected out and the theory is non-perturbative and strongly coupled. Second, the asymmetric $\mathbb{Z}_{4}$ will also act on the transversal graviton G-field; we will call this transformation G-transformation, which is very similar to Ehlers transformations in General Relativity. Since the massless graviton is not-invariant under the G-transformation, it will be projected out as well, and the theory does not contain any physical graviton state anymore. Hence we call this theory a topological string theory. Note that the combined action on the 
S-field and on the G-field becomes an element of the Geroch group. It is nothing else than a T-duality transformation, when further going down from four to two dimensions, as it was discussed in [6]. However unlike in [6], we have to augment in $4 \mathrm{D}$ string theory the dilaton and metric transformations by a third type of projection, that we denote it by H-transformation, and which acts on the helicity degrees of freedom and ensures four-dimensional Lorentz-invariance. This $\mathrm{H}$-transformation was not performed in [6], with the result that the two-dimensional theories in [6] in general posses chiral $(\mathcal{P}, \mathcal{Q})$ supersymmetry and are not up-liftable to a four-dimensionional Lorentz-invariant theory. We will comment on this interplay between four and two dimensions more below. So upshot of this discussion will be that the combined action of S- and G- and H-transformations will correspond to the S-duality transformations of supersymmetric field theory, which act on the supercharges in the way as described in section 2.3

Before we start the explicit discussion, let us also mention that in contrast to the two-dimensional models, for which a full modular invariant partition functions can be constructed [6], the non-perturbative, four-dimensional string models do not allow for an immediate construction of a one-loop partition function. Furthermore we will also not consider twisted sectors, where in two dimensions additional supercharges have emerged in the completely left-right asymmetric orbifold constructions. Here will will only discuss the $\mathbb{Z}_{4}$ invariant, but untwisted sector of the theory. At the same time we will assume here that possible twisted sectors do not provide any further supercharges in four dimensions.

\subsection{R-symmetry and T-duality twist}

The implementation of the $\mathbb{Z}_{4}$ R-symmetry rotation is relatively straightforward and we will follow the construction in [6]. Here we will give a few more details in terms of the non-geometric interpretation as T-fold. The full T-duality group for the heterotic string on $T^{6}$ is given by the discrete group $\mathrm{SO}(6,22 ; \mathbb{Z})$. For our purpose it is enough to work out the action of the various target space duality transformations on the internal string coordinates of $T^{6}=\prod_{K=1}^{3} T_{i}^{2}$. The relevant T-duality subgroup of $\operatorname{SO}(6,22 ; \mathbb{Z})$ is given by the following modular group:

$$
G=O(2,2 ; \mathbb{Z})^{3}=\prod_{K=1}^{3}\left[\mathrm{SL}(2)_{\tau_{K}} \times \mathrm{SL}(2)_{\rho_{K}} \times \mathbb{Z}_{2}^{\tau_{K} \leftrightarrow \rho_{K}} \times \mathbb{Z}_{2}^{\tau_{K} \leftrightarrow-\bar{\rho}_{K}}\right] .
$$

Here the $\rho_{K}$ and $\tau_{K}$ are the three Kähler respectively complex structure moduli of the three subtori $T_{K}^{2}$.

Let us first restrict the discussion to one particular subtorus. Particular $O(2,2)$ transformations act as in general left-right asymmetric transformations on the internal left- and right-moving coordinates $Y_{L}^{i}(\bar{z})$ and $Y_{R}^{i}(z)(i=1,2)$ of $T^{2}$ :

$$
\vec{Y}_{L} \rightarrow \mathcal{M}_{L} \vec{Y}_{L}, \quad \vec{Y}_{R} \rightarrow \mathcal{M}_{R} \vec{Y}_{R}
$$

In the following we are interested in those $\mathcal{M}_{\mathrm{SO}(2,2)}$ transformations, for which the group elements $\left(\mathcal{M}_{L}, \mathcal{M}_{R}\right)$ correspond to discrete rotations $\mathbb{Z}_{N}^{L} \times \mathbb{Z}_{M}^{R}$ on the torus coordinates. 
This is possible, provided that a faithful embedding of $\mathbb{Z}_{N}^{L} \times \mathbb{Z}_{M}^{R}$ into $O(2,2)$ can be found (see [32] for more details). Introducing a complex coordinate on the torus,

$$
Z(z, \bar{z})=Y^{1}(z, \bar{z})+i Y^{2}(z, \bar{z})=Z_{L}(\bar{z})+Z_{R}(z),
$$

the $O(2,2)$ transformations then act as particular discrete rotations on the complexified left- and right-moving coordinates:

$$
Z_{L}(\bar{z}) \rightarrow e^{2 i \pi / N_{L}} Z_{L}(\bar{z}), \quad Z_{R}(z) \rightarrow e^{2 i \pi / N_{R}} Z_{R}(z)
$$

Next consider the corresponding right-moving, complex world sheet fermions $\Psi^{K}$. Their transformation behavior can be deduced from the requirement that the right-moving world sheet supersymmetry commutes with the target space modular transformations. This requirement follows from the fact that the action of the right-moving supercurrent connects equivalent (picture-changed) physical string states. The right-moving (internal) world sheet supercurrent has the form

$$
T_{F}(z)=\sum_{K=1}^{3}\left(\Psi_{R}^{K} \partial \bar{Z}_{R}^{K}+\bar{\Psi}_{R}^{K} \partial Z_{R}^{K}\right)(z) .
$$

Demanding the supercurrent to be invariant under modular transformations, one derives that the complex fermions $\Psi(z)$ of each subtorus transform only under the rotations of the right-movers as

$$
\Psi(z) \rightarrow e^{2 i \pi / N_{R}} \Psi(z) .
$$

It follows that these transformations act on the three two-dimensional bosons $H$ as

$$
H(z) \rightarrow H(z)+2 \pi / N_{R}
$$

One can consider three particular cases: (i) $N_{L}=N_{R} \neq 1$, (ii) $N_{L}=-N_{R} \neq 1$ and (iii) $N_{L}=1, N_{R} \neq 1$. Case (i), the symmetric rotation, corresponds to a transformation, which only acts on the complex structure modulus $\tau$ but not on the Kähler modulus $\rho$. Conversely the asymmetric case (ii) can be realized by T-duality transformations that act only on $\rho$ but not on $\tau$. Finally, the completely asymmetric case (iii), for which the leftmoving degrees of freedom are inert, corresponds to transformations which simultaneously act on $\rho$ and on $\tau$. For the reasons explained above, this is the case of interest for us, and to be specific we choose $-N_{L}=N_{R}=4$. In order to realize this asymmetric projection one has to freeze the moduli to their self-dual values, i.e. $\tau=\rho=i$. It follows that the corresponding moduli fields are projected out of the physical spectrum of the string.

Now we are ready to examine the modular transformation properties of the space-time supercharges $Q^{\alpha, A}$ in eqs. (3.7) and (3.8). Namely they transform with a particular common phase under these T-duality transformations. Specifically we obtain that the supercharges in the right-moving sector of the heterotic string transform in the same way as:

$$
T \text { - duality : } \quad Q^{\alpha, A} \rightarrow e^{-\frac{i \pi\left(w_{1}^{A}+w_{2}^{A}+w_{3}^{A}\right)}{2}} Q^{\alpha, A} .
$$


These transformations agree with the R-transformations in field theory, described above (see eq. (2.12)). In fact, the discrete $\mathbb{Z}_{4}$ elements of the T-duality group act like discrete R-symmetry transformations, which are subgroups of the $\mathrm{SO}(6)$ automorphism group of the $\mathcal{N}=4$ supersymmetry algebra. Hence the charges of the space-time fields under the target space modular transformation are closely related to their corresponding R-charges, as it was already discussed in $[47,48]$

\subsection{S-duality twist}

S-duality: S-duality [33] exists in the four-dimensional heterotic string and also in the NS sector of the type II strings as non-perturbative symmetry that acts on the four-dimensional dilaton-axion field, denoted by $S=a+i e^{-2 \Phi}$, as $\mathrm{SL}(2, \mathbb{Z})_{S}$ transformations ( $\Phi$ is the dilaton field and $a$ is the dualized four-dimensional $B_{\mu \nu}$ field):

$$
S \rightarrow \frac{a S+b}{c S+d}, \quad a d-b c=1
$$

In the four-dimensional effective field theory, the non-perturbative S-duality transformations map the perturbative heterotic states with electric charges onto the non-perturbative states with magnetic charges and vice versa. S-duality also maps the electromagnetic field strengths and the dual field strength into each other. From a ten-dimensional string point of view, S-duality exchanges the elementary strings with the solitonic five-branes. During the compactification process on $T^{6}$ one is wrapping the five-branes around the internal 5-cycles, and then the electric-magnetic S-duality in four dimensions becomes manifest.

World-sheet action of S-duality: in addition to its non-perturbative action, S-duality also acts on the world-sheet fields in a particular, non-trivial way, which will be important for the construction of the S-folds. For that purpose let us introduce also a complex coordinate for the uncompactified, transversal coordinates $X^{1}$ and $X^{2}$ :

$$
Z_{L}^{0}(\bar{z})=X^{1}(\bar{z})_{L}+i X^{2}(\bar{z})_{L}, \quad Z_{R}^{0}(z)=X^{1}(z)_{R}+i X^{2}(z)_{R}
$$

Then the complex S-field is associated to the following vertex operator:

$$
\text { S - field : } S=\bar{\partial} \bar{Z}_{L}^{0}(\bar{z}) \Psi^{0}(z) e^{-\phi(z)} .
$$

As already observed in [33], this operator is in complete analogy to the marginal operator of the internal Kähler modulus $\rho$ of an internal 2-torus. Indeed, from the world-sheet conformal field theory point of view, the modes $\rho$ and $S$ are very similar, both corresponding to $\bar{\partial} Z_{L} \partial Z_{R}$ marginal deformations with respect to the compactified or uncompactified spatial coordinates (with periodic boundary conditions in the internal directions). The action of the S-duality transformations have the same left-right asymmetric effect on the uncompactified transversal coordinates $Z_{L}^{0}$ and $Z_{R}^{0}$ and world-sheet fermions as the modular T-duality transformations on the Kähler modulus $\rho$ have on the internal world-sheet fields. In fact if we further compactified the two transverse coordinates $Z^{0}$ and $\bar{Z}^{0}$ on a torus, the 
parallelism between $\rho$ and $S$ would be strict, but of course, that is now not the case. ${ }^{8}$ The fact, that S-duality is reduced to T-duality when putting the theory on a 2-torus was also discussed in the context of four-dimensional $\mathcal{N}=4$ super Yang-Mills theory [49].

World-sheet action of G-duality: as already advertised, in addition to S-duality we introduce a another kind of duality transformation, which we call G-duality. It acts on the two light-cone degrees of freedom of the graviton field in uncompactified four-dimensional Minkowski space. G-duality transformations act on the world-sheet fields $Z_{L}^{0}$ and $Z_{R}^{0}$ in a left-right-symmetry way, in complete analogy to the action of $\operatorname{SL}(2)_{\tau}$ duality transformations on the internal world-sheet fields of a 2-torus. Therefore, in analogy to the complex structure modulus $\tau$ on the compact two-torus, let us combine the two transversal degrees of freedom of the four-dimensional graviton field into one complex graviton field $G$ as follows:

$$
G=\frac{g_{12}}{g_{11}}+i \frac{\sqrt{\operatorname{det} g}}{g_{11}} .
$$

Now $\mathrm{SL}(2)_{g}$ acts on this field in the standard way:

$$
G \rightarrow \frac{a G+b}{c G+d}, \quad a d-b c=1 .
$$

Seen from the point of general relativity, the G-duality transformations are just a certain kind of large diffeomorphisms. Namely they act like finite, four-dimensional coordinate transformations. From the world-sheet point of view they have the same left-right symmetric action on the uncompactified transversal coordinates and world-sheet fermions as the corresponding modular T-duality transformations on the complex modulus $\tau$ have on the internal world-sheet fields of a compact two-torus.

Combined world-sheet action of S-duality and G-duality: like on the two-torus, it also becomes evident that $\mathrm{SL}(2)_{S}$ and $\mathrm{SL}(2)_{G}$ combine into the group $O(2,2)_{S, G} \simeq$ $\mathrm{SL}(2)_{S} \times \mathrm{SL}(2)_{G}$. This group is a symmetry of the general relativity and also of the twodimensional string $\sigma$-model, in case the four-dimensional background possesses two Killing symmetries. The group $O(2,2)_{S, G}$ is the socalled Geroch group [50] and the string Geroch group was already discussed in $[51,52]$ some time ago. Moreover, it follows that group elements of the rank seven group $O(6,6) \times \mathrm{SL}(2)_{S}$ are promoted by the inclusion of $\mathrm{SL}(2)_{G}$ to certain group elements of the rank eight group $O(8,8)$. This group is identical to the T-duality group of $T^{8}$, when we compactify to two dimensions. In general S- and G-duality transformations act the uncompactified, transversal world-sheet fields as

$$
Z_{L}^{0}(\bar{z}) \rightarrow e^{2 i \pi / M_{L}} Z_{L}^{0}(\bar{z}), \quad Z_{R}^{0}(z) \rightarrow e^{2 i \pi / M_{R}} Z_{R}^{0}(z)
$$

and

$$
\Psi^{0}(z) \rightarrow e^{2 i \pi / M_{R}} \Psi^{0}(z) .
$$

\footnotetext{
${ }^{8}$ So compared to F-theory, where S-duality originates from the modular transformations on the complex structure of the auxiliary type IIB torus, the heterotic S-duality can be seen to have its origin from the "torus" that describe the transversal spatial coordinates in four dimensions.
} 
We can now consider again the three different cases, depending how S-duality and Gduality transformations act on the left- and right-moving complex coordinate $Z_{L}^{0}$ and $Z_{R}^{0}$. (i) $M_{L}=M_{R} \neq 1$, (ii) $M_{L}=-M_{R} \neq 1$ and (iii) $M_{L}=1, M_{R} \neq 1$. Case (i), the symmetric rotation, corresponds to a transformation, which only acts on the metric $G$ but not on the S-field. Conversely the asymmetric case (ii) can be realized by S-duality transformations that act only on $S$ but not on $G$. Finally, the completely asymmetric case (iii), for which the left-moving degrees of freedom are inert, corresponds to transformations which simultaneously act on $S$ and on $G$. Therefore we call it Geroch twist. Again like for the T-duality transformations, this is the case of interest for us, and to be specific we choose $-M_{L}=M_{R}=4$. In order to realize this asymmetric projection one has to freeze the fields to their self-dual values, i.e. $S=G=i$. It follows that the massless graviton as well as the S-field is projected out of the physical spectrum

It now follows that the eight heterotic space-time supercharges in eq. (3.7) and in eq. (3.8) transform, depending on their helicity, with an opposite phase factor under S-Gduality transformations, namely

$$
\text { Geroch twist } \equiv(S \otimes G)-\text { duality }: \quad Q^{\alpha=\frac{1}{2}, A} \rightarrow e^{\frac{i \pi}{4}} Q^{\alpha=\frac{1}{2}, A} .
$$

and

$$
\text { Geroch twist } \equiv(S \otimes G)-\text { duality }: \quad Q^{\alpha=-\frac{1}{2}, A} \rightarrow e^{-\frac{i \pi}{4}} Q^{\alpha=-\frac{1}{2}, A} .
$$

This is almost agrees with the action of S-duality in field theory. However we realize that this transformation acts on the supercharges in a non Lorentz-invariant way. Namely comparing this transformation with the transformation of the supercharges in field theory, as given in eq. (2.13), we see that they do not agree with each other: whereas in field theory, the S-duality phase factor does not depend on the spinor $\alpha$, the string construction so far leads to opposite phases for the two spinor weights $\alpha= \pm \frac{1}{2}$. Combining it with T-duality the number of conserved supercharges is only six instead of twelve, as required by four-dimensional Lorentz invariance.

The H-twist: as already announced, in order to ensure Lorentz-invariance of the Sduality transformations, we will have to augment the S-G-duality transformations, by a further twist operation, denoted by $H$, which acts differently on two components of the fermionic spinor degrees of freedom and reverses the phase factor of the positive helicity spinors:

$$
H-\text { twist }: \quad Q^{\alpha=\frac{1}{2}, A} \rightarrow e^{-\frac{i \pi}{2}} Q^{\alpha=\frac{1}{2}, A}, \quad Q^{\alpha=-\frac{1}{2}, A} \rightarrow Q^{\alpha=-\frac{1}{2}, A} .
$$

The S-G-H twist is an automorphism in string theory and hence it is an allowed projection. It acts as left-right-asymmetric rotation on the transversal coordinate. The charges of all fields under the S-G-H twist are same as the charges under the discrete Sduality transformation in field theory. Therefore the upshot of this discussion is that what we have considered as S-duality in $\mathcal{N}=4$ supersymmetric field theories is represented in string theory as the product of $S$ and $H$ transformations. If one in addition requires to act only the right-moving world-sheet fields, one also has to include the G-transformations:

$$
\mathrm{S}-\text { duality in } \mathcal{N}=4 \text { field theory } \equiv S \otimes G \otimes H \text { in string theory }
$$


Note that the H-twist was not performed in [6]. There only the S-G rotations on the left- and right moving coordinates were performed, which become identical to T-duality transformations when going to two dimensions. Without only the S-G rotations being performed, the S-fold breaks 4D-Lorentz invariance. However in two-dimensions this is not a problem, and it results in a chiral $2 \mathrm{D}$ theories with $(\mathcal{P}, \mathcal{Q})$ supersymmetry.

\section{Massive spectrum of $\mathrm{W}$-superstrings}

Now we finally proceed to examine the spectrum of some string theory S-folds. Their spectra are given by those states which are invariant under the combined discrete T-SG-H rotations. The graviton $G^{(i j)}$, the antisymmetric tensor $B^{[i j]}$ and the dilaton $\Phi$ are invariant under the T-duality transformations. Other fields may transform with a particular phase, since the contain either internal world-sheet bosons or internal world sheet fermions in the vertex operators. On the other hand the vertex operators of the graviton $G^{(i j)}$, the antisymmetric tensor $B^{[i j]}$ and the dilaton $\Phi$ are not invariant under the S-G-H-transformations. Only fields with only internal degrees of freedom are invariant under S-duality transformations. Hence when building the heterotic or type II S-folds with $\mathcal{N}=3$ or $\mathcal{N}=7$ supersymmetry, the entire massless perturbative spin-two supermultiplet is projected out of the string spectrum. Only massive string excitations can survive the S-fold projection. Therefore it is very plausible that the non-perturbative $\mathcal{N}=7$ supergravity, which arises as effective theory from the string theory, is the massive, higher spin W-supergravity theory, which we have discussed before.

In the four-dimensional effective field theory, non-perturbative S-duality transformations map the perturbative heterotic states with electric charges onto the non-perturbative states with magnetic charges and vice versa. After the $\mathbb{Z}_{4}$ projection, the S-field is frozen to its self-dual value $S=i$ and hence the string coupling constant is fixed at strong coupling. It follows that in the S-fold, the non-perturbative electric and magnetic states have equal masses, which are comparable with the masses of the perturbative massive string states. In the following we will nevertheless neglect the non-perturbative states in the discussion of the spectrum of the S-folds, but we will only derive the spectrum of the massive excited, perturbative string states of the four-dimensional heterotic and type II S-folds that survive the $\mathbb{Z}_{4}$ projection. We will restrict the discussion on the states at the first massive string level. In particular we will construct the massive spectrum of $\mathcal{N}=3$ and $\mathcal{N}=7$ type II W-strings.

\subsection{Massive states of the unprojected four-dimensional fermionic string with $\mathcal{N}=4$ supersymmetry}

We will start by recalling the massive spectrum of the (left or right-moving) fermionic string after the GSO projection, but before projecting on the $\mathbb{Z}_{4}$ invariant states. These states will be needed later on, when discussing the $\mathcal{N}=3,7$ S-folds. 
Bosons: splitting the indices into uncompactified and internal indices, one obtains at the first massive level the following massive states (see for example [53]):

$$
\begin{array}{llll}
b_{-1 / 2}^{i} b_{-1 / 2}^{j} b_{-1 / 2}^{I}|0\rangle, & b_{-1 / 2}^{i} b_{-1 / 2}^{I} b_{-1 / 2}^{J}|0\rangle, & b_{-1 / 2}^{I} b_{-1 / 2}^{J} b_{-1 / 2}^{K}|0\rangle, & \\
b_{-3 / 2}^{i}|0\rangle, & b_{-3 / 2}^{I}|0\rangle & & \\
\alpha_{-1}^{i} b_{-1 / 2}^{j}|0\rangle, & \alpha_{-1}^{i} b_{-1 / 2}^{I}|0\rangle, & \alpha_{-1}^{I} b_{-1 / 2}^{i}|0\rangle, & \alpha_{-1}^{I} b_{-1 / 2}^{J}|0\rangle .
\end{array}
$$

(Here the $b$ 's and the $\alpha$ 's are the oscillators of the world-sheet fermions and bosons.) Collecting all states and putting them into proper massive representations of the fourdimensional little group $\mathrm{SO}(3)$ as well as in proper representations of the $\mathcal{N}=4 \mathrm{SU}(4)$ R-symmetry, one obtains the following massive spectrum:

$$
\underline{1} \times \operatorname{Spin}(2)+(\underline{6}+\underline{6}+\underline{15}) \times \operatorname{Spin}(1)+\left(2 \times \underline{1}+\underline{10}+\underline{10}+\underline{20^{\prime}}\right) \times \operatorname{Spin}(0) .
$$

As discussed before, for massive states in $\mathcal{N}=4$ supersymmetry the R-symmetry group is enhanced from $\mathrm{U}(4)$ to $\mathrm{USp}(8) \supset \mathrm{U}(4)$ with the following branching rules:

$$
\begin{aligned}
\underline{8} & =\underline{4}+\underline{\overline{4}}, \\
\underline{27} & =\underline{6}+\underline{6}+\underline{15}, \\
\underline{36} & =\underline{1}+\underline{10}+\underline{10}+\underline{15}, \\
\underline{42} & =2 \times \underline{1}+\underline{10}+\underline{10}+\underline{20^{\prime}}, \\
\underline{48} & =\underline{4}+\underline{\overline{4}}+\underline{20}+\underline{20}
\end{aligned}
$$

Then the massive bosons transform under $\operatorname{USp}(8)$ as

$$
\underline{1} \times \operatorname{Spin}(2)+(\underline{27}) \times \operatorname{Spin}(1)+(\underline{42}) \times \operatorname{Spin}(0) .
$$

Fermions: in ten dimensions, the 128 massive fermions are given by the following string states:

$$
(8)_{c}+(56)_{c}: \quad b_{-1}^{A}|a\rangle, \quad(8)_{s}+(56)_{s}: \quad \alpha_{-1}^{A}|\dot{a}\rangle .
$$

In terms of four-dimensional massive spinors this leads to:

$$
(\underline{4}+\underline{\overline{4}}) \times \operatorname{Spin}(3 / 2)+(\underline{4}+\underline{\overline{4}}+\underline{20}+\underline{20}) \times \operatorname{Spin}(1 / 2),
$$

where in this decomposition each spin 3/2 Rarita Schwinger field in four dimensions contains 4 degrees of freedom and each spin 1/2 Dirac fermion possess 2 degrees of freedom. Under USp(8) the massive fermions transform as

$$
(\underline{8}) \times \operatorname{Spin}(3 / 2)+(\underline{48}) \times \operatorname{Spin}(1 / 2) \text {, }
$$

The bosons in eq. (4.2) together with the fermions in eq. (4.7) build one long, massive $\mathcal{N}=4$ spin 2 supermultiplet. It precisely agrees with the super Weyl multiplet $W_{\mathcal{N}=4}^{2}$, which is displayed in eq. (2.20). 


\subsection{Massive states of the $\mathbb{Z}_{4}$-projected four-dimensional fermionic string with $\mathcal{N}=3$ supersymmetry}

Now we perform the $\mathbb{Z}_{4}$ S-fold projection on the above massive spectrum of the rightmoving sector of the fermionic string. Then supersymmetry is broken from $\mathcal{N}=4$ to $\mathcal{N}=3$, the R-symmetry is broken from $\mathrm{SU}(4)$ to $\mathrm{U}(3)$ and the supercharge transforms as $\underline{3}$ under the R-symmetry. We emphasize again that the $\mathrm{H}$-projection must be taken into account in order to get the correct Lorenz-invariant spectrum.

Bosons: the 64 invariant states can be grouped into the massive representations of the four-dimensional little group $\mathrm{SO}(3)$ and of the $\mathrm{SU}(3)$ R-symmetry. But for the massive states in $\mathcal{N}=3$ supersymmetry, the R-symmetry group is enhanced from $\mathrm{U}(3)$ to $\mathrm{USp}(6) \supset$ $\mathrm{U}(3)$ with the following branching rules:

$$
\begin{aligned}
\underline{6} & =\underline{3}+\underline{\overline{3}}, \\
\underline{14} & =\underline{3}+\underline{\overline{3}}+\underline{8} .
\end{aligned}
$$

Then the massive bosons transform under $\operatorname{USp}(6)$ as

$$
\operatorname{Spin}(2)+(\underline{14}+\underline{1}) \times \operatorname{Spin}(1)+\underline{14} \times \operatorname{Spin}(0) .
$$

Fermions: acting with the $\mathbb{Z}_{4}$ transformation on the spinor fields, the invariant states build the following 64 massive fermions in four dimensions

$$
\underline{6} \times \operatorname{Spin}(3 / 2)+\left(\underline{14^{\prime}}+\underline{6}\right) \times \operatorname{Spin}(1 / 2) .
$$

Note that the bosons in eq. (4.9) together with the fermions in eq. (4.10) build one massive $\mathcal{N}=3$ spin-two supermultiplet, which perfectly agrees with the spin-two super Weyl multiplet $W_{\mathcal{N}=3}$, as given in eq. (2.17).

\subsection{Massive states of the bosonic string}

For completeness we also need the massive states of the left-moving bosonic string, when we build the heterotic string. Leaving out the internal gauge coordinates, we get the following 44 massive states in ten dimensions:

$$
(8)_{v}: \quad \alpha_{-2}^{A}|0\rangle, \quad(35)_{0}+1_{0}: \quad \alpha_{-1}^{A} \alpha_{-1}^{B}|0\rangle .
$$

They correspond to the following massive bosonic states in four dimensions:

$$
\operatorname{Spin}(2)+6 \times \operatorname{Spin}(1)+21 \times \operatorname{Spin}(0) .
$$

The massive spin-two field is universal and will be present in any background. The 6 massive vectors and the 21 massive scalars can be regarded as additional matter fields, which appear in case the internal left-moving is sector is given by a six-dimensional torus. 


\section{$4.4 \mathcal{N}=3$ heterotic $\mathrm{W}$-superstring}

This non-perturbative heterotic theory is given by the tensor product of the right-moving massive spectrum of section 4.2 times the left-moving spectrum of section 4.3. Seen as field theory double construction, it corresponds to the $\mathcal{N}=3$, W-supergravity, which is a tensor product $\operatorname{QFT}(\mathcal{N}=3) \otimes \operatorname{QFT}(\mathcal{N}=0)$. The universal sector of the $\mathcal{N}=3 \mathrm{~W}$ supergravity is obtained by building the tensor product of the left-moving massive spin-two field in eq. (4.12) with the right-moving $\mathcal{N}=3$ Weyl multiplet: the massive spectrum has the following form:

$$
\begin{array}{ll}
\text { B : } & {[\operatorname{Sppin}(2)+15 \times \operatorname{Spin}(1)+14 \times \operatorname{Spin}(0)]_{R} \times[\operatorname{Spin}(2)]_{L},} \\
\text { F }: & {[6 \times \operatorname{Spin}(3 / 2)+20 \times \operatorname{Spin}(1 / 2)]_{R} \times[\operatorname{Spin}(2)]_{L} .}
\end{array}
$$

Explicitly performing the tensor product this leads to the following spectrum of massive fermions and bosons:

$$
\begin{array}{ll}
\mathrm{B}: & {[\operatorname{Spin}(4)+16 \times \operatorname{Spin}(3)+30 \times \operatorname{Spin}(2)+16 \times \operatorname{Spin}(1)+\operatorname{Spin}(0)]} \\
\mathrm{F}: & {[6 \times \operatorname{Spin}(7 / 2)+26 \times \operatorname{Spin}(5 / 2)+26 \times \operatorname{Spin}(3 / 2)+6 \times \operatorname{Spin}(1 / 2)] .}
\end{array}
$$

These states build two long, massive $\mathcal{N}=3$ supermultiplets, namely one $(j=5 / 2)$ with top spin-four $\left(n_{B}+n_{F}=6 \times 64=384\right)$ plus another one $(j=3 / 2)$ with top spin-three $\left(n_{B}+n_{F}=4 \times 64=256\right)$, where the multiplicities of these states fall into representations of the group $\mathrm{USp}(6) \supset \mathrm{U}(3)$. It contains in total $n_{B}+n_{F}=640$ degrees of freedom.

If one also includes the additional six spin-one and 21 spin-zero fields of the left-moving bosonic string in eq. (4.12) into account, the massive spectrum has the following form:

$$
\begin{gathered}
\text { B : } \quad[\operatorname{Spin}(2)+15 \times \operatorname{Spin}(1)+14 \times \operatorname{Spin}(0)]_{R} \\
\times[\operatorname{Spin}(2)+6 \times \operatorname{Spin}(1)+21 \times \operatorname{Spin}(0)]_{L}, \\
\text { F : } \quad[6 \times \operatorname{Spin}(3 / 2)+20 \times \operatorname{Spin}(1 / 2)]_{R} \\
\times[\operatorname{Spin}(2)+6 \times \operatorname{Spin}(1)+21 \times \operatorname{Spin}(0)]_{L} .
\end{gathered}
$$

Then the tensor product takes following form:

$$
\begin{array}{ll}
\text { B : } & {[\operatorname{Spin}(4)+22 \times \operatorname{Spin}(3)+147 \times \operatorname{Spin}(2)+511 \times \operatorname{Spin}(1)+385 \times \operatorname{Spin}(0)]} \\
\text { F : } & {[6 \times \operatorname{Spin}(7 / 2)+62 \times \operatorname{Spin}(5 / 2)+308 \times \operatorname{Spin}(3 / 2)+582 \times \operatorname{Spin}(1 / 2)]}
\end{array}
$$

These states build a reducible massive $\mathcal{N}=3$ spin-four supermultiplet, and it contains in total $n_{B}=n_{F}=2816$ degrees of freedom.

\section{5 $\mathcal{N}=7$ type II $\mathrm{W}$-superstring}

The $\mathcal{N}=7=4_{L}+3_{R}$ non-perturbative type II $\mathrm{W}$-superstring is given as the product of a left-moving times a right-moving fermionic string with four respectively three spacetime supercharges. It is strongly coupled and it does not contain any massless fields. The 
massive sector is given by the tensor product of the right-moving massive spectrum of section 4.2 times the left-moving spectrum of section 4.1 in the following way:

$$
\begin{aligned}
& \text { B : } \quad[\operatorname{Spin}(2)+15 \times \operatorname{Spin}(1)+14 \times \operatorname{Spin}(0)]_{R} \\
& \times[\operatorname{Spin}(2)+27 \times \operatorname{Spin}(1)+42 \times \operatorname{Spin}(0)]_{L}, \\
& {[6 \times \operatorname{Spin}(3 / 2)+20 \times \operatorname{Spin}(1 / 2)]_{R}} \\
& \times[8 \times \operatorname{Spin}(3 / 2)+48 \times \operatorname{Spin}(1 / 2)]_{L} \\
& \text { F : } \quad[6 \times \operatorname{Spin}(3 / 2)+20 \times \operatorname{Spin}(1 / 2)]_{R} \\
& \times[\operatorname{Spin}(2)+27 \times \operatorname{Spin}(1)+42 \times \operatorname{Spin}(0)]_{L}, \\
& {[\operatorname{Spin}(2)+15 \times \operatorname{Spin}(1)+14 \times \operatorname{Spin}(0)]_{R}} \\
& \times[8 \times \operatorname{Spin}(3 / 2)+48 \times \operatorname{Spin}(1 / 2)]_{L} .
\end{aligned}
$$

Altogether we explicitly derive in this way the following $\mathcal{N}=7$ spectrum of massive fermions and bosons:

$$
\begin{aligned}
& \text { B : } \quad[\operatorname{Spin}(4)+91 \times \operatorname{Spin}(3)+1000 \times \operatorname{Spin}(2)+2912 \times \operatorname{Spin}(1)+2002 \times \operatorname{Spin}(0)], \\
& \text { F : } \quad\left[14 \times \operatorname{Spin}\left(\frac{7}{2}\right)+364 \times \operatorname{Spin}\left(\frac{5}{2}\right)+1988 \times \operatorname{Spin}\left(\frac{3}{2}\right)+3068 \times \operatorname{Spin}\left(\frac{1}{2}\right)\right] .
\end{aligned}
$$

These states build exactly one long, massive $\mathcal{N}=7$ spin-four supermultiplet. It perfectly agrees with the massive, spin-four multiplet in eq. (2.24), which we have obtained via the double copy construction of $\mathcal{N}=7 \mathrm{~W}$-supergravity and where the multiplicities of these states fall into representations of the group $\operatorname{USp}(14) \supset \mathrm{U}(7)$. The agreement of the string theory construction and the field theory double copy construction provides some convincing evidence that $\mathcal{N}=7 \mathrm{~W}$-supergravity indeed exists as physical theory. Since the theory does not contain any massless fields, and hence in particular also no massless gravitons and also no massless dilaton, it should be strongly coupled and also be a kind of topological theory, since there are no possible fluctuations around a given gravitational background.

\section{Conclusions and outlook}

In this paper we have provided evidence for the existence of new $\mathrm{W}$-supergravity and $\mathrm{W}$-superstring theories. They are built as double copy constructions involving the nonperturbative $\mathcal{N}=3$ supersymmetric Yang-Mills theories, or in the string context, containing a right-moving fermionic string with $\mathcal{N}=3$ space-time supersymmetry. In particular we have obtained in this way a massive, spin-four W-supergravity/superstring with $\mathcal{N}=7$ (28 supercharges) supersymmetry in four space-time dimensions. This theory can be regarded as a non-perturbative S-fold of $\mathcal{N}=8$ supergravity or, respectively, of the $\mathcal{N}=8$ type II superstring theory.

The proposed $\mathcal{N}=7$ string construction possesses the following three distinct features:

- The internal space is a non-geometric background, obtained by modding out with the T-duality group. This eliminates all internal massless moduli. 
- The discrete S-duality transformation corresponds to left-right asymmetric rotation of the uncompactified transversal coordinates. Therefore also the uncompactified space can be regarded as non-geometric background. Then, along the uncompactified directions, the S-duality is modded out. Therefore the theory is strongly coupled without a massless dilaton.

- In four dimensions there is an additional twist by large diffeomorphisms. Therefore there is no massless graviton multiplet, and the theory is a massive, topological theory. Together with the S-duality, this twist corresponds to a discrete element of the Geroch group. Note that the Geroch group also contains a kind of mirror symmetry in the uncompactified transversal directions, namely the exchange of the S-field with the (transversal) graviton field.

As a caveat we like to mention that we have discarded possible twisted sectors in our string construction, we have just performed a truncation of the entire string spectrum on the states, which are invariant under the discrete asymmetric rotations. Due to their nonperturbative character, we also could not construct a modular invariant partition function for the $\mathrm{W}$-superstrings. Therefore there is at the moment no proof that the $\mathrm{W}$-superstrings are fully consistent, full fledged string theories.

We also like to emphasize that, although being massive theories, $\mathrm{W}$-supergravities are locally supersymmetric theories. It would be very interesting to learn more about the structure of these theories. Their (effective) description is not in terms of a Lagrangian but only in terms of their operator content, their massive fields and their on-shell scattering amplitudes. Hence relevant information might be obtained by computing string scattering amplitudes with massive external fields, as it was done e.g. in [54, 55].

Finally we like to comment about a possible relation between the holographic picture and the double copy picture of supergravity theories. This follows from the intriguing correspondence between the $4 \mathrm{D} \mathcal{N}$-extended Weyl multiplets and the $5 \mathrm{D}$ standard $2 \mathcal{N}$ extended supergravity multiplets. This is because they both deal with $4 \mathrm{D}$ massive spin-2 respresentations of the same superconformal algebra. In this way, the $4 \mathrm{D}$ superconformal algebra of $\mathcal{N}$-extended quantum field theories $(\mathcal{N} \leq 4)$ at the four-dimensional boundary is holographically equivalent to the supersymmetry algebra of $2 \mathcal{N}$-extended $A d S_{5}$ supergravity theories. However the difference between this two cases is that for the double copy construction the spin-two field lives on the $4 \mathrm{D}$ boundary, whereas in the holographic duality the spin-two fields lives in the 5D bulk space. Following this observation, if we identify Weyl supergravity with the superconformal $\mathcal{N}=4$ boundary theory, then its holographic dual should be an $\mathcal{N}=8$ a spin-four $\mathrm{W}$-supergravity theory (for comprehensive reviews and some papers on higher spin theories see [56-59]). Note that four-dimensional Weyl supergravity violates unitarity do to the quartic derivative action which leads to a dipole ghost [60-63]. It is therefore an interesting question if this also implies a lack of unitarity in the spin-four $\mathrm{W}$ supergravity.

\section{Acknowledgments}

We like to thank Inaki Garcia-Etxebarria, Wolfgang Lerche, Diego Regalado and in particular Ioannis Florakis for very useful discussions. The work of S.F. is supported in part 
by CERN TH Dept and INFN-CSN4-GSS. The work of D.L. is supported by the ERC Advanced Grant "Strings and Gravity" (Grant No. 320045) and the Excellence Cluster Universe. He also is grateful to the CERN theory department for its hospitality, when part of this work was performed.

\section{A Supercharges}

Starting from ten dimensions, the 16 supercharges $Q^{\alpha, A}$ and $Q^{\dot{\alpha}, \dot{A}}$ belong to the 16 dimensional spinor representation $\underline{16}_{s}$ of the group $\mathrm{SO}(10)$. Decomposing this group into the four-dimensional Lorentz-group times the R-symmetry group, i.e. $\mathrm{SO}(10) \supset$ $\mathrm{SO}(1,3) \times \mathrm{SO}(6)_{R}$, the spinor $\underline{16}_{S}$ decomposes as

$$
Q^{\alpha, A}, Q^{\dot{\alpha}, \dot{A}}: \quad \underline{16}_{s}=\left(\underline{2}_{s}, \underline{4}_{s}\right)+\left(\underline{2}_{c}, \underline{4}_{c}\right) .
$$

Further we also need the 10-dimensional transversal Lorentz-group $\mathrm{SO}(8)_{T}$, which is the little group for massless states, and under which the spinor of $\mathrm{SO}(10) \supset \mathrm{SO}(8)_{T} \times \mathrm{SO}(2)_{L}$ decomposes as

$$
\underline{16}_{s}=\left(\underline{8}_{s}, \frac{1}{2}\right)+\left(\underline{8}_{c},-\frac{1}{2}\right) .
$$

Here $\mathrm{SO}(2)_{L}$ is the rotation group, which acts on the longitudinal degrees of freedom of the massless states.

Finally we need also the branching of $\mathrm{SO}(8)_{T}$ into the four-dimensional, transversal little group $\mathrm{SO}(2)_{T}$ times the R-symmetry group $\mathrm{SO}(6)_{R}$, i.e. $\mathrm{SO}(8)_{T} \supset \mathrm{SO}(2)_{T} \times \mathrm{SO}(6)_{R}$ :

$$
\underline{8}_{s}=\left(\frac{1}{2}, \underline{4}_{s}\right)+\left(-\frac{1}{2}, \underline{4}_{c}\right), \quad \underline{8}_{c}=\left(-\frac{1}{2}, \underline{4}_{s}\right)+\left(\frac{1}{2}, \underline{4}_{c}\right) .
$$

Comparing the different decompositions, one sees that the eight supercharges $Q^{\alpha, A}$ belong to the two first representations in $\underline{8}_{s}$ and $\underline{8}_{c}$, namely

$$
Q^{\alpha, A}: \quad\left(\frac{1}{2}, \underline{4}_{s}\right)+\left(-\frac{1}{2}, \underline{4}_{s}\right)
$$

whereas the other eight supercharges $Q^{\dot{\alpha}, \dot{A}}$ belong to the two second representations in $\underline{8}_{s}$ and $\underline{8}_{c}$, namely

$$
Q^{\dot{\alpha}, \dot{A}}: \quad\left(\frac{1}{2}, \underline{4}_{c}\right)+\left(-\frac{1}{2}, \underline{4}_{c}\right),
$$

Open Access. This article is distributed under the terms of the Creative Commons Attribution License (CC-BY 4.0), which permits any use, distribution and reproduction in any medium, provided the original author(s) and source are credited.

\section{References}

[1] D.Z. Freedman, P. van Nieuwenhuizen and S. Ferrara, Progress toward a theory of supergravity, Phys. Rev. D 13 (1976) 3214 [INSPIRE]. 
[2] S. Deser and B. Zumino, Consistent supergravity, Phys. Lett. B 62 (1976) 335 [InSPIRE].

[3] A. Salam and E. Sezgin, Supergravities in diverse dimensions, North Holland/Word Scientific, The Netherlands (1989).

[4] D.Z. Freedman and A. Van Proeyen, Supergravity, Cambridge University Press, Cambridge U.K. (2012).

[5] B. de Wit, A.K. Tollsten and H. Nicolai, Locally supersymmetric $D=3$ nonlinear $\sigma$-models, Nucl. Phys. B 392 (1993) 3 [hep-th/9208074] [INSPIRE].

[6] I. Florakis, I. Garcia-Etxebarria, D. Lüst and D. Regalado, $2 d$ orbifolds with exotic supersymmetry, JHEP 02 (2018) 146 [arXiv: 1712.04318] [INSPIRE].

[7] I. Garcia-Etxebarria and D. Regalado, $\mathcal{N}=3$ four dimensional field theories, JHEP 03 (2016) 083 [arXiv: 1512.06434] [INSPIRE].

[8] O. Aharony and M. Evtikhiev, On four dimensional $N=3$ superconformal theories, JHEP 04 (2016) 040 [arXiv: 1512.03524] [INSPIRE].

[9] O. Aharony and Y. Tachikawa, S-folds and $4 d N=3$ superconformal field theories, JHEP 06 (2016) 044 [arXiv : 1602.08638] [INSPIRE].

[10] S. Ferrara, M. Porrati and A. Zaffaroni, $N=6$ supergravity on $A d S_{5}$ and the $\mathrm{SU}(2,2 / 3)$ superconformal correspondence, Lett. Math. Phys. 47 (1999) 255 [hep-th/9810063] [INSPIRE].

[11] Z. Bern, J.J.M. Carrasco and H. Johansson, New relations for gauge-theory amplitudes, Phys. Rev. D 78 (2008) 085011 [arXiv:0805.3993] [INSPIRE].

[12] Z. Bern, T. Dennen, Y.-t. Huang and M. Kiermaier, Gravity as the square of gauge theory, Phys. Rev. D 82 (2010) 065003 [arXiv: 1004.0693] [INSPIRE].

[13] Z. Bern, J.J.M. Carrasco and H. Johansson, Perturbative quantum gravity as a double copy of gauge theory, Phys. Rev. Lett. 105 (2010) 061602 [arXiv:1004.0476] [INSPIRE].

[14] A. Anastasiou et al., Yang-Mills origin of gravitational symmetries, Phys. Rev. Lett. 113 (2014) 231606 [arXiv: 1408.4434] [INSPIRE].

[15] L. Borsten and M.J. Duff, Gravity as the square of Yang-Mills?, Phys. Scripta 90 (2015) 108012 [arXiv: 1602 .08267] [INSPIRE].

[16] G.L. Cardoso, S. Nagy and S. Nampuri, A double copy for $\mathcal{N}=2$ supergravity: a linearised tale told on-shell, JHEP 10 (2016) 127 [arXiv:1609.05022] [INSPIRE].

[17] A. Anastasiou et al., Are all supergravity theories Yang-Mills squared?, arXiv:1707.03234 [INSPIRE].

[18] S. Ferrara, M. Kaku, P.K. Townsend and P. van Nieuwenhuizen, Gauging the graded conformal group with unitary internal symmetries, Nucl. Phys. B 129 (1977) 125 [INSPIRE].

[19] E. Bergshoeff, M. de Roo and B. de Wit, Extended conformal supergravity, Nucl. Phys. B 182 (1981) 173 [INSPIRE].

[20] J. van Muiden and A. Van Proeyen, The $\mathcal{N}=3$ Weyl multiplet in four dimensions, arXiv: 1702.06442 [INSPIRE].

[21] B. de Wit, J.W. van Holten and A. Van Proeyen, Transformation rules of $N=2$ supergravity multiplets, Nucl. Phys. B 167 (1980) 186 [INSPIRE].

[22] S. Ferrara and B. Zumino, Transformation properties of the supercurrent, Nucl. Phys. B 87 (1975) 207 [INSPIRE]. 
[23] S. Ferrara and B. Zumino, Structure of conformal supergravity, Nucl. Phys. B 134 (1978) 301 [INSPIRE].

[24] B. de Wit and S. Ferrara, On higher order invariants in extended supergravity, Phys. Lett. B 81 (1979) 317.

[25] H. Kawai, D.C. Lewellen and S.H.H. Tye, A relation between tree amplitudes of closed and open strings, Nucl. Phys. B 269 (1986) 1 [INSPIRE].

[26] S. Stieberger, Open $\mathcal{G}$ closed vs. pure open string disk amplitudes, arXiv:0907.2211 [INSPIRE].

[27] S. Stieberger and T.R. Taylor, New relations for Einstein-Yang-Mills amplitudes, Nucl. Phys. B 913 (2016) 151 [arXiv:1606.09616] [InSPIRE].

[28] D. Lüst and S. Theisen, Exceptional groups in string theory, Int. J. Mod. Phys. A 4 (1989) 4513 [INSPIRE].

[29] S. Ferrara, D. Lüst and S. Theisen, World sheet versus spectrum symmetries in heterotic and Type II superstrings, Nucl. Phys. B 325 (1989) 501 [INSPIRE].

[30] S. Ferrara and C. Kounnas, Extended supersymmetry in four-dimensional type II strings, Nucl. Phys. B 328 (1989) 406 [inSPIRE].

[31] C. Condeescu, I. Florakis and D. Lüst, Asymmetric orbifolds, non-geometric fluxes and non-commutativity in closed string theory, JHEP 04 (2012) 121 [arXiv:1202.6366] [INSPIRE].

[32] C. Condeescu, I. Florakis, C. Kounnas and D. Lüst, Gauged supergravities and non-geometric Q/R-fluxes from asymmetric orbifold CFT's, JHEP 10 (2013) 057 [arXiv:1307.0999] [INSPIRE].

[33] A. Font, L.E. Ibáñez, D. Lüst and F. Quevedo, Strong-weak coupling duality and nonperturbative effects in string theory, Phys. Lett. B 249 (1990) 35 [INSPIRE].

[34] J.H. Schwarz and A. Sen, Duality symmetries of $4 D$ heterotic strings, Phys. Lett. B 312 (1993) 105 [hep-th/9305185] [INSPIRE].

[35] A. Sen, Strong-weak coupling duality in four-dimensional string theory, Int. J. Mod. Phys. A 9 (1994) 3707 [hep-th/9402002] [INSPIRE].

[36] S. Ferrara, J. Scherk and B. Zumino, Algebraic properties of extended supergravity theories, Nucl. Phys. B 121 (1977) 393 [inSPIRE].

[37] D.Z. Freedman, SO(3) invariant extended supergravity, Phys. Rev. Lett. 38 (1977) 105 [INSPIRE].

[38] S. Ferrara, C.A. Savoy and B. Zumino, General massive multiplets in extended supersymmetry, Phys. Lett. B 100 (1981) 393.

[39] M. Kaku, P.K. Townsend and P. van Nieuwenhuizen, Superconformal unified field theory, Phys. Rev. Lett. 39 (1977) 1109 [INSPIRE].

[40] M. Kaku, P.K. Townsend and P. van Nieuwenhuizen, Properties of conformal supergravity, Phys. Rev. D 17 (1978) 3179 [inSPIRE].

[41] E.S. Fradkin and A.A. Tseytlin, Conformal supergravity, Phys. Rept. 119 (1985) 233 [INSPIRE].

[42] H. Johansson and A. Ochirov, Pure gravities via color-kinematics duality for fundamental matter, JHEP 511 (2015) 046 [arXiv:1407.4772]. [INSPIRE]. 
[43] H. Johansson, G. Kälin and G. Mogull, Two-loop supersymmetric QCD and half-maximal supergravity amplitudes, JHEP 09 (2017) 019 [arXiv: 1706.09381] [INSPIRE].

[44] N. Berkovits and E. Witten, Conformal supergravity in twistor-string theory, JHEP 08 (2004) 009 [hep-th/0406051] [INSPIRE].

[45] R. Kallosh, Cancellation of conformal and chiral anomalies in $\mathcal{N} \geq 5$ supergravities, Phys. Rev. D 95 (2017) 041701 [arXiv: 1612.08978] [INSPIRE].

[46] D. Lüst, T-duality and closed string non-commutative (doubled) geometry, JHEP 12 (2010) 084 [arXiv: 1010.1361] [INSPIRE].

[47] L.E. Ibáñez and D. Lüst, Duality anomaly cancellation, minimal string unification and the effective low-energy Lagrangian of $4 D$ strings, Nucl. Phys. B 382 (1992) 305 [hep-th/9202046] [INSPIRE].

[48] L.E. Ibáñez and D. Lüst, A comment on duality transformations and (discrete) gauge symmetries in four-dimensional strings, Phys. Lett. B 302 (1993) 38 [hep-th/9212089] [INSPIRE].

[49] J.A. Harvey, G.W. Moore and A. Strominger, Reducing $S$ duality to T duality, Phys. Rev. D 52 (1995) 7161 [hep-th/9501022] [INSPIRE].

[50] R.P. Geroch, A method for generating new solutions of Einstein's equation. 2, J. Math. Phys. 13 (1972) 394 [INSPIRE].

[51] I. Bakas, $O(2,2)$ transformations and the string Geroch group, Nucl. Phys. B 428 (1994) 374 [hep-th/9402016] [INSPIRE].

[52] M.J. Duff, Strong/weak coupling duality from the dual string, Nucl. Phys. B 442 (1995) 47 [hep-th/9501030] [INSPIRE].

[53] R. Blumenhagen, D. Lüst and S. Theisen, Basic concepts of string theory, Springer, Germany (2012).

[54] W.-Z. Feng et al., Direct production of lightest Regge resonances, Nucl. Phys. B 843 (2011) 570 [arXiv: 1007.5254] [INSPIRE].

[55] W.-Z. Feng, D. Lüst and O. Schlotterer, Massive supermultiplets in four-dimensional superstring theory, Nucl. Phys. B 861 (2012) 175 [arXiv:1202.4466] [INSPIRE].

[56] X. Bekaert, S. Cnockaert, C. Iazeolla and M.A. Vasiliev, Nonlinear higher spin theories in various dimensions, hep-th/0503128 [INSPIRE].

[57] L.F. Alday and J.M. Maldacena, Comments on operators with large spin, JHEP 11 (2007) 019 [arXiv: 0708.0672] [INSPIRE].

[58] M. Henneaux and S.-J. Rey, Nonlinear $W_{\infty}$ as asymptotic symmetry of three-dimensional higher spin Anti-de Sitter gravity, JHEP 12 (2010) 007 [arXiv: 1008.4579] [INSPIRE].

[59] A. Sagnotti, Notes on strings and higher spins, J. Phys. A 46 (2013) 214006 [arXiv:1112.4285] [INSPIRE].

[60] K.S. Stelle, Renormalization of higher derivative quantum gravity, Phys. Rev. D 16 (1977) 953 [INSPIRE].

[61] K.S. Stelle, Classical gravity with higher derivatives, Gen. Rel. Grav. 9 (1978) 353 [INSPIRE].

[62] K.S. Stelle, Abdus Salam and quadratic curvature gravity: classical solutions, Int. J. Mod. Phys. A 32 (2017) 1741012 [INSPIRE].

[63] L. Álvarez-Gaumé et al., Aspects of quadratic gravity, Fortsch. Phys. 64 (2016) 176 [arXiv: 1505.07657] [INSPIRE]. 